\title{
Amodal Processing in Human Prefrontal Cortex
}

\author{
Benjamin J. Tamber-Rosenau, ${ }^{1,2}$ Paul E. Dux, ${ }^{3}$ Michael N. Tombu, ${ }^{4}$ Christopher L. Asplund, ${ }^{5}$ and René Marois ${ }^{1,2}$ \\ ${ }^{1}$ Department of Psychology, and ${ }^{2}$ Vanderbilt Vision Research Center, Vanderbilt University, Nashville, Tennessee 37240, ${ }^{3}$ School of Psychology, University \\ of Queensland, Brisbane 4072, Queensland, Australia, ${ }^{4}$ Defence Research and Development Canada Toronto, Toronto, Ontario M3K 2C9, Canada, and \\ ${ }^{5}$ Division of Social Sciences, Yale-NUS College, Singapore 138614
}

Information enters the cortex via modality-specific sensory regions, whereas actions are produced by modality-specific motor regions. Intervening central stages of information processing map sensation to behavior. Humans perform this central processing in a flexible, abstract manner such that sensory information in any modality can lead to response via any motor system. Cognitive theories account for such flexible behavior by positing amodal central information processing (e.g., "central executive," Baddeley and Hitch, 1974; "supervisory attentional system," Norman and Shallice, 1986; "response selection bottleneck," Pashler, 1994). However, the extent to which brain regions embodying central mechanisms of information processing are amodal remains unclear. Here we apply multivariate pattern analysis to functional magnetic resonance imaging (fMRI) data to compare response selection, a cognitive process widely believed to recruit an amodal central resource across sensory and motor modalities. We show that most frontal and parietal cortical areas known to activate across a wide variety of tasks code modality, casting doubt on the notion that these regions embody a central processor devoid of modality representation. Importantly, regions of anterior insula and dorsolateral prefrontal cortex consistently failed to code modality across four experiments. However, these areas code at least one other task dimension, process (instantiated as response selection vs response execution), ensuring that failure to find coding of modality is not driven by insensitivity of multivariate pattern analysis in these regions. We conclude that abstract encoding of information modality is primarily a property of subregions of the prefrontal cortex.

\section{Introduction}

Perception and performance rely upon modality-specific brain mechanisms at the input (sensory) and output (motor) stages of processing. In part because these peripheral stages can be flexibly mapped to one another-information in any sensory modality can drive output in any motor modality-cognitive theories posit intervening stage(s) of amodal central processing (e.g., Baddeley and Hitch, 1974; Norman and Shallice, 1986; Pashler, 1994; Baddeley, 2003). The observation that tasks differing in both sensory and motor modalities can interfere with one another (e.g., Pashler et al., 1993; Pashler, 1994; Kamienkowski et al., 2011) supports this view. However, it is unclear how amodal central stages are instantiated in the brain. Recent fMRI studies have described broad "task-positive" (TP) network(s) (Fox et al., 2005; Vincent et al., 2008; Gao and Lin, 2012) that largely match "multiple demand" (MD) or "adaptive coding" networks (Duncan and Owen, 2000; Duncan, 2001, 2010), frontoparietal regions activated across a wide range of neuroimaging studies of cognition. Given that these

\footnotetext{
Received Sept. 27, 2012; revised May 24, 2013; accepted May 30, 2013.

Author contributions: B.J.T.-R., P.E.D., M.N.T., C.L.A., and R.M. designed research; B.J.T.-R., P.E.D., M.N.T., and C.L.A. performed research; B.J.T.-R. analyzed data; B.J.T.-R. and R.M. wrote the paper.

This work was supported by National Institutes of Health Grant R01 MH 70776 to R.M., and Grants 5 T32 EY 007135 and P30 EY008126 to the Vanderbilt Vision Research Center, as well as Australian Research Council Australian Postdoctoral Fellowship DP0986387 and Future Fellowship FT120100033 to P.E.D. We thank Samir Zughni and Nikhil Kulkarni for excellent technical assistance.

The authors declare no competing financial interests.

Correspondence should be addressed to Dr. Benjamin J. Tamber-Rosenau, Department of Psychology, Vanderbilt University, 627 Wilson Hall, 111 21st Avenue South, Nashville, TN 37240. E-mail: benjamin.j.tamberrosenau@vanderbilt.edu.

DOI:10.1523/JNEUROSCI.4601-12.2013

Copyright $\odot 2013$ the authors $\quad 0270-6474 / 13 / 3311573-15 \$ 15.00 / 0$
}

regions are recruited across very diverse tasks, they may represent loci of amodal, abstract information processing. Regions of the lateral prefrontal cortex (LPFC), including TP/MD regions, appear to be particularly good candidates because existing theoretical frameworks posit that LPFC is organized along a continuum from less to more abstract representations of information along its posterioranterior axis (Fuster, 2001; Koechlin et al., 2003; Badre, 2008; Badre and D'Esposito, 2009). However, it remains an open question whether sensory or motor modalities are represented individually or in an amodal, integrated manner at each of these levels of abstraction.

Past searches for amodal brain regions assessed fMRI activation overlap across tasks in different modalities (Szameitat et al., 2002; Jiang and Kanwisher, 2003; Dux et al., 2006; Marois et al., 2006; Dux et al., 2009; Ivanoff et al., 2009; Schumacher et al., 2011; Tombu et al., 2011), primarily using response selection (RS) paradigms because RS is often thought to rely on amodal operations (Pashler, 1994; Kamienkowski et al., 2011). These previous studies revealed multiple cortical sites, many of which substantially overlap the TP/MD networks. However, the low spatial resolution of $\mathrm{fMRI}$ coupled with univariate analyses did not allow them to distinguish whether different modalities engage the same neural ensembles within a brain region or whether they instead recruit distinct but adjacent ensembles. Hence, mere overlap of activation does not guarantee that identical neural ensembles process information regardless of modality.

This resolution limitation can be largely alleviated by multivariate pattern analysis (MVPA). MVPA is sensitive to within-region spatial activation patterns that reflect differences at the supravoxel (Op de Beeck, 2010a,b) or subvoxel level (Kamitani and Tong, 2005; 
Kamitani and Sawahata, 2010). Thus, MVPA has greater power than univariate fMRI for testing whether identical neural ensembles are recruited by distinct modalities. In the present study, we used MVPA to test whether brain regions involved in RS are insensitive to the joint modalities of sensory input and motor output, as predicted of amodal regions that use abstract information codes.

\section{Materials and Methods}

Participants. Experiments 1 (Dux et al., 2009), 2 (Tombu et al., 2011), and 3 (Dux et al., 2006) relied upon previously published data; participant details are described in their respective publications. Briefly, these experiments contained 7, 12, and 9 participants, respectively. Experiment 4 contained data from 6 participants ( 5 male, 1 female, mean age 26.11 years, SEM 2.02 years); data were not examined from 3 additional participants (all female) because of eyetracker and audio presentation equipment failures and from 2 additional participants (both male) because of failure to follow task instructions. Experiment 5 used data from 10 participants (6 male, 4 female, mean age 29.20 years, SEM 1.67 years) from a previously unpublished section of a doctoral dissertation (Asplund, 2010); data from one additional subject were excluded because of failure to follow task instructions.

All participants gave informed consent as approved by the Vanderbilt University Institutional Review Board.

Tasks and stimuli. The paradigms used in Experiments 1-4 each consisted of two arbitrary stimulus-response mapping tasks (Fig. 1A, Experiment 4 task) that were distinct in their sensory input (either auditory or visual) and motor output (manual, vocal, or oculomotor) modalities. In each experiment, sensory and motor modality were confounded; thus, our conclusions can speak to coding of modality in general but are not specific to sensory input system or motor output system. Experiment 5 (Fig. 1B, the Experiment 5 task) used a different task that separated in time the task phases of perceptual selection, response selection, and motor execution. The key methodological features of Experiments 1-3 are described below, and greater methodological detail is presented in their respective original publications. Experiments 4 and 5 are described fully here because they have not appeared in previous papers. Details of fMRI acquisition and run number and duration for all experiments are presented in Table 1.

In Experiment 1, there were two possible complex auditory stimuli (one complex tone and one edited natural sound) that each required a distinct speeded vocal response and two possible visual stimuli (faces) that each required a distinct speeded manual response. Vocal responses consisted of uttering the syllables "Tay" or "Koo"; manual responses consisted of pressing buttons with either the right index or middle finger. Trials began with a 200 ms visual cue (an enlarged fixation point); the cue was immediately followed by a $200 \mathrm{~ms}$ stimulus presentation. Twelve seconds elapsed before the onset of the next trial. Trial types in Experiment 1 were randomly ordered. Experiment 1 also included dual-task trials to allow comparison of dual- and single-task activations (Dux et al., 2009); data from these trials were not examined in the present analysis. Subjects performed Experiment 1 in three separate fMRI sessions so that training effects could be assessed; the present analysis pools data across the three sessions.

Experiment 2 used an identical paradigm to Experiment 1 with the following exceptions: there were three possible stimuli and responses in
Face $200 \mathrm{msec}$

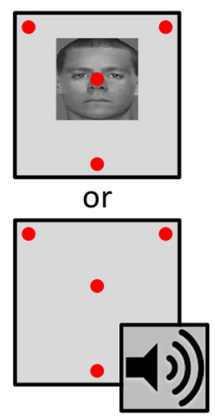

Fixation +

Sound

$200 \mathrm{msec}$
Saccadic

Response

$+\mid \mathrm{TI}$

$13.5 \mathrm{sec}$

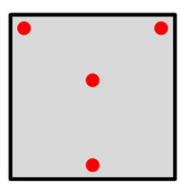

Manual

Response

$+\mid \mathrm{TI}$

$13.5 \mathrm{sec}$

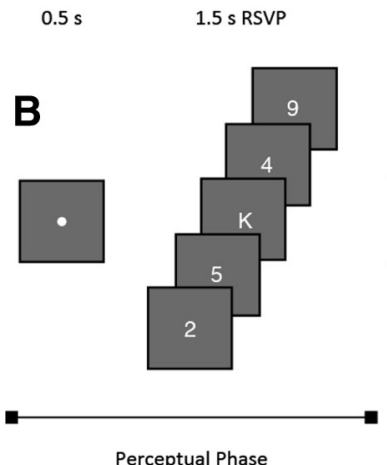

$14 \mathrm{~s}$

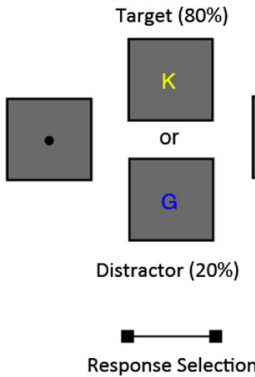

Phase

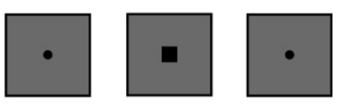

Response Execution Phase

Figure 1. A, Experimental design for Experiment 4. In one task, visually presented face stimuli were mapped to oculomotor saccade responses. In another task, auditory complex sound stimuli were mapped to manual button-press responses. Any given trial contained one wo tasks. Experiments 1-3 used similar paradigms with two arbitrary stimulus-response mapping tasks (for details, see Materials S s delay, the RS phase followed. In the RS phase, the color of a target-matching letter was mapped to a manual motor response to be executed later. After another $14 \mathrm{~s}$ delay, the RE phase was cued by enlargement of the fixation point.

each modality, the intertrial interval was $14 \mathrm{~s}$, and all data were collected in a single fMRI session.

The stimulus-response modality mappings from Experiments 1 and 2 were reversed in Experiment 3: the eight possible auditory stimuli each required a distinct manual response, and the eight possible color patch stimuli each required a distinct vocal response. The experiment used a rapid event-related design: trials occurred with an exponential distribution of trial onset asynchronies $(8,6.4,4.8$, or $3.2 \mathrm{~s})$. Trial types were randomly ordered, and there were no dual-task trials.

In Experiment 4, there were three possible auditory stimuli that each required distinct manual responses (right index, middle, or ring finger) and three possible face stimuli (squares with sides of 4.5 degrees; presented at central fixation) that each required location-specific saccades. Saccade destinations were equidistant locations on the perimeter of an imaginary circle centered on fixation (radius, 8.3 degrees) and were marked by red dots (diameter, 0.3 degrees) that remained on screen throughout the experiment, except during block instruction periods. These marked saccade destinations were to the upper left, upper right, or directly below fixation. The fixation point was identical to the saccade target dots. All stimuli were presented on a gray background. Trials consisted of a $200 \mathrm{~ms}$ visual cue (doubled diameter of fixation point) followed after $100 \mathrm{~ms}$ by stimulus presentation for $200 \mathrm{~ms}$. Behavioral responses were collected during the first $3.5 \mathrm{~s}$ of the $13.5 \mathrm{~s}$ intertrial interval. Trials in Experiment 4 were organized into six blocks of six trials each per run; each block contained trials of only one stimulus and response modality. Blocks began with a $4.5 \mathrm{~s}$ instruction period consisting of either "SOUNDS" or "FACES" presented centrally, followed by 14 s of fixation before the first trial of the block. 
Table 1. fMRI acquisition parameters for Experiments 1-5

\begin{tabular}{|c|c|c|c|c|c|c|c|c|c|c|c|}
\hline \multirow[b]{2}{*}{ Experiment } & \multicolumn{2}{|l|}{ Runs } & \multirow[b]{2}{*}{$\mathrm{TR}(\mathrm{ms})$} & \multirow[b]{2}{*}{ FOV (mm) } & \multicolumn{2}{|c|}{ Matrix (mm) } & \multicolumn{3}{|l|}{ Slices } & \multicolumn{2}{|l|}{ Voxel size (mm) } \\
\hline & Number & Duration (s) & & & Acquired & Reconstructed & Number & Thickness (mm) & Gap (mm) & Reconstructed & Resampled \\
\hline 1 & $24(8 / d)$ & 300 & 2000 & 240 & $80 \times 80$ & $128 \times 128$ & 33 & 3.5 & 0.5 & $1.875 \times 1.875 \times 4$ & $3 \times 3 \times 3$ \\
\hline 4 & $6-8$ & 612 & 2000 & 192 & $64 \times 64$ & $64 \times 64$ & 35 & 3 & 0 & $3 \times 3 \times 3$ & $3 \times 3 \times 3$ \\
\hline 5 & 8 & 288 & 2000 & 240 & $80 \times 80$ & $128 \times 128$ & 33 & 3 & 0.5 & $1.875 \times 1.875 \times 3.5$ & $3 \times 3 \times 3$ \\
\hline
\end{tabular}

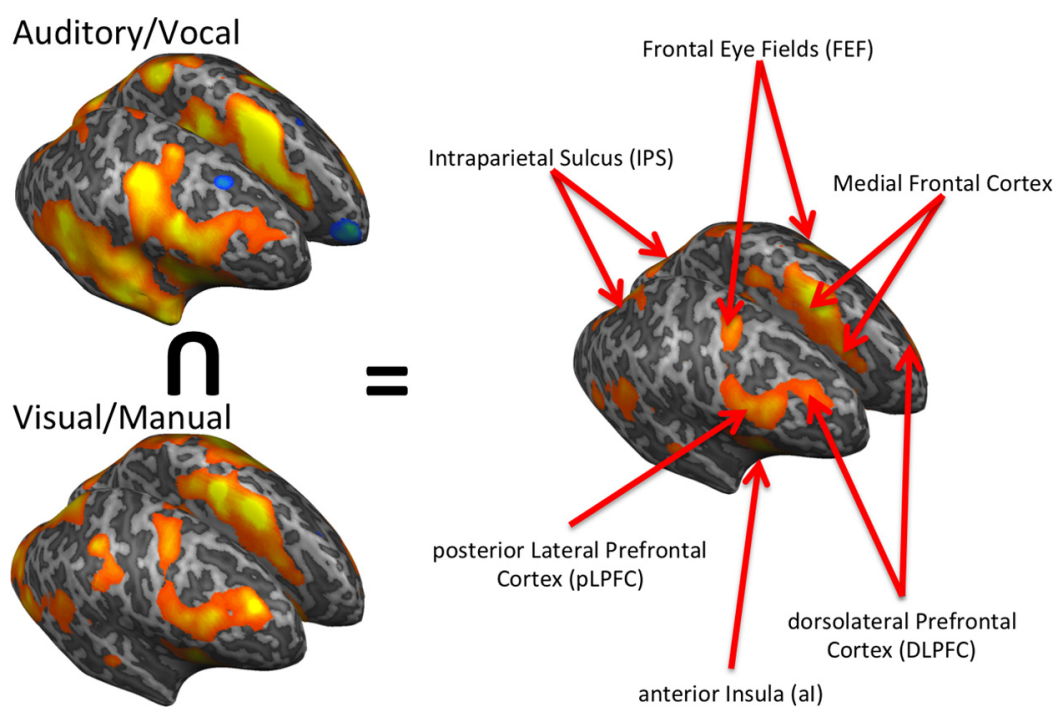

Figure 2. ROIs sensitive to RS tasks across sensory and motor modalities. The ROls were isolated in Experiment 2 by taking the conjunction of the open contrasts of auditory/vocal and visual/manual trials to yield a group random-effects map. Left, The open contrast was calculated for each sensory/motor pairing. Right, The conjunction of these contrasts was the basis for region selection.

Instead of comparing across modalities within the same task process (RS), Experiment 5 contrasted two cognitive processes (Fig. 1B): RS and response execution (RE). All stimuli in Experiment 5 were presented on a gray background. In an initial perceptual selection phase, subjects viewed a $500 \mathrm{~ms}$ cue (fixation dot change from black to white) immediately followed by a 15 item, $1.5 \mathrm{~s}$ duration RSVP stream of digits presented at fixation (100 ms per item, no temporal gap, white Helvetica font, 0.8 degrees character size, including the numbers 2-9, randomly ordered with replacement, with the constraint that no digit could appear twice in any three-frame temporal window). A single letter (the target) replaced the digit in one of frames $5-13$. This letter was to be remembered over a $14 \mathrm{~s}$ delay (fixation-only display) until the RS phase, in which a probe letter was presented centrally for $2 \mathrm{~s}$. If this probe letter matched the memorized letter (termed "valid" trials; $\sim 80 \%$ of trials overall), subjects then chose a manual response (one of six button presses using either index, middle, or ring finger) based upon the color of the probe (blue, purple, green, yellow, cyan, red). If the probe did not match the target ("invalid trial"), subjects were to ignore its color, withhold all responses, and simply fixate through the remainder of the trial. After an additional 14 s fixation-only display, the RE phase began with a cue (slightly enlarged fixation with a change in shape from circular to square; $2 \mathrm{~s}$ duration); subjects executed their manual responses upon presentation of this cue or ignored the cue and made no response on invalid trials. Subjects completed six trials in each run of Experiment 5.

fMRI acquisition. All MRI data were acquired on 3T Philips Intera Achieva scanners at the Vanderbilt University Institute of Imaging Science. In all experiments, structural MRIs were acquired using conventional parameters, with $1 \mathrm{~mm}^{3}$ resolution covering the entire brain. Functional scans in all experiments used echo-planar imaging and were acquired with an ascending-interleaved order of axial slices. The methodology for fMRI data acquisition for Experiments 1-3 is described in detail in their respective original reports, although key acquisition parameters for all experiments are summarized in Table 1. Two runs of data from a single subject in Experiment 4 were not analyzed because of audio presentation equipment failure.

Stimulus presentation and response collection. With the exception of Experiment 4, all visual stimuli were presented using LCD projectors on a screen at the head of the bore of the magnet and viewed via a mirror mounted to the head coil. In Experiment 4, stimuli were viewed using Nordic NeuroLaboratory VisualSystem goggles with $800 \times 600$ pixels of resolution. Auditory stimuli were presented via MRI-compatible headphones. All stimulus presentation and response collection were accomplished using MATLAB (MathWorks) and the Psychophysics Toolbox (Brainard, 1997; Pelli, 1997; Kleiner et al., 2007).

Manual responses were collected using 5-key keypads (one per hand, where appropriate; Rowland Institute of Science, Cambridge, MA). Vocal responses were collected using a Commander XG MR-compatible headset (which also served for auditory presentation in Experiments 1-3). Oculomotor responses in Experiment 4 were collected via the Nordic NeuroLaboratory goggles add-on EyeTracking Camera system coupled to Arrington ViewPoint software. Custom software written in MATLAB was used to baseline eyetracking coordinates to trial onset, filter for blinks and other artifacts, find fixations (when 3 successive samples from the eyetracker were within $\sim 1$ degree of visual angle of one another), and match fixations to approximate saccade target destinations. To determine these matches, fixations were included within an ellipse such that the nominal saccade destination was one focus of the ellipse, the major axis of the ellipse was oriented along the line passing from central fixation through the nominal saccade destination, and the closest approach of the ellipse to fixation was $\sim 4.2$ degrees of eccentricity (i.e., halfway between fixation and the nominal saccade destination).

$f M R I$ preprocessing. All data were processed using a combination of BrainVoyager QX version 2.2, BVQX Tools (J. Weber, Columbia University, New York; available at http://support.brainvoyager.com/ available-tools/52-matlab-tools-bvxqtools.html), LIBSVM (Chang and Lin, 2011) with the OSU-SVM MATLAB interface (J. Ma, Y. Zhao, S. Ahalt, D. Eads; available at http://svm.sourceforge.net/download.shtml), and custom MATLAB code. Data from Experiments 1-3 were additionally processed using ANTs (Avants et al., 2011) and Tools for NIfTI (J. Shen, Rotman Research Institute, Toronto, Ontario; available at http://www. rotman-baycrest.on.ca/ jimmy/).

For all experiments, data were subjected to slice acquisition time correction, rigid-body motion correction, and linear trend removal in BrainVoyager. Temporal high-pass filtering was conducted for Experiments 4 ( 4 cycles per run) and 5 ( 3 cycles per run) to correct for possible drift in baseline signal between blocks (Experiment 4) or trial phases (Experiment 5). Data were spatially smoothed with a Gaussian kernel of $6 \mathrm{~mm}$ FWHM before GLM analysis in BrainVoyager, but nonsmoothed data were used for MVPA analysis with LIBSVM. All data were warped to Talairach space (Talairach and Tournoux, 1988) in BrainVoyager because recent research has indicated that the minor spatial smoothing 
Table 2. Comparison of conjunction regions to multiple demand and task positive networks ${ }^{a}$

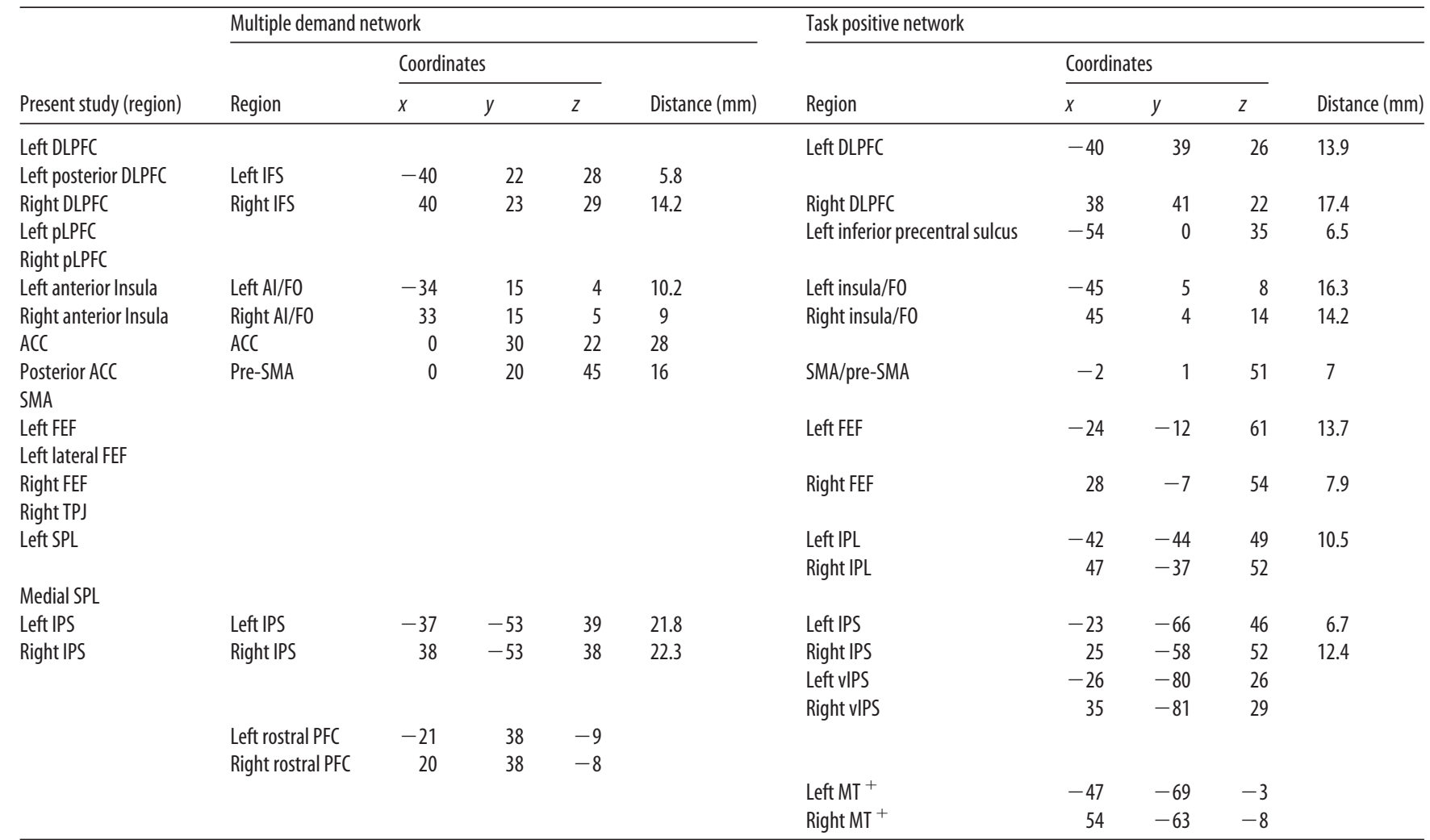

${ }^{a}$ Each row represents a single brain region or activation. Multiple demand regions are drawn from Duncan (2010; their Fig. 1). Coordinates were converted from MNI to Talairach space using the online Yale Biolmage Suite nonlinear MNI to Talairach Coordinate Converter (available at http://www.bioimagesuite.org/Mni2Tal/index.html), which accounts for the small hemispheric asymmetries despite the originally symmetric MNI coordinates. Task positive regions are drawn from Fox et al. (2005; their Table 1). Distance reflects millimeters from the specified coordinates to the peak activation of the closest region from the present study (see Table 3 for the present study's coordinates). Because all fMRI activations have extensive spread around the peaks, there is likely substantial overlap between our ROIs and those of the task positive and multiple demand networks. The multiple demand network coordinates also served as the centers of ROl spheres for the follow-up analysis of canonical multiple demand peak coordinates.

inherent in the warping and resampling process does not impair and may aid MVPA (Op de Beeck, 2010a,b; Etzel et al., 2011). At the stage of Talairach warping, all data were resampled into BrainVoyager's default 3 $\mathrm{mm}$ isotropic functional voxel space.

Data in Experiments 1-3 were additionally warped to a common functional template, using a method differing from but following the basic logic of Sabuncu et al. (2010) to maximize power to detect weak MVPA classification (see below). The goal of functional warping is to reduce the possibility that variability across subjects in the assignment of functional processes to fine-structure brain anatomy could contribute to null results. Thus, functional warping helps to rule out a trivial cause for our null findings. However, because functional warping is not a standard processing step, we ensured that our results were not contingent upon its use by excluding it from Experiments 4 and 5 . Additionally, we directly compared the effect of functional warping within the same dataset by reanalyzing Experiment 2 (as it had the greatest number of participants) using identical ROIs (here, those derived from the functionally warped data; see below), but with nonfunctionally warped, nonspatially smoothed Talairach-warped data. This reanalysis left the results of Experiment 2 largely unchanged: when we compared MVPA performance with and without functional warping using a global paired $t$ test on regional MVPA-versus-artifact $t$ scores, omitting warping yielded a nonsignificant decrease in sensitivity $\left(t_{(17)}=\right.$ $-0.439, p=0.793)$.

Functional warping was accomplished using ANTs for morph calculations and application. Tools for NIfTI were used for the transformation between ANTs' required NIfTI format and BrainVoyager's proprietary VTC format for 4-dimensional fMRI data. Functional warping began by calculating a morph between the unthresholded statistical $t$-map of the conjunction (Nichols et al., 2005) of each subject's open contrast of all RS events without regard for modality (see fMRI analysis: univariate GLM) to the map from a single subject from Experiment 1, which served as a template. This morph was then applied to each volume of preprocessed functional imaging data, which was finally reassembled into a BrainVoyager 4-dimensional fMRI data file (VTC format) for further analysis.

Univariate GLM. For Experiments 1-4, fMRI data were subjected to separate univariate GLM analyses (Friston et al., 1995) in which, in addition to nuisance regressors (e.g., dual-task trials for Experiments 1 and 2), separate regressors were modeled for correct trials in each perceptual and motor modality. Thus, each experiment yielded two regressors of interest (e.g., auditory/vocal and visual/manual trials in Experiment 1). We calculated the conjunction (Nichols et al., 2005) of the open contrasts of each of these regressors to identify all voxels sensitive to the performance of a RS task in both of the perceptual and motor modalities tested in each experiment (Ivanoff et al., 2009).

Experiment 5 was subjected to an identical analysis, except that the regressors of interest were valid-trial RS and RE phases (with separate regressors for left- and right-handed RE); nuisance regressors included the perceptual selection phase, invalid trial RS phase, and errors/absent responses. We then calculated the conjunction of the open contrasts of valid trial RS and RE to identify voxels sensitive to both processes.

ROIs. To standardize the results, the same set of ROIs was probed across all five experiments. These ROIs were defined in Experiment 2 because it had the greatest number of participants (an initial analysis of Experiment 1 using Experiment 1-derived ROIs yielded qualitatively similar results to using the Experiment 2 ROIs). Although the multivariate comparison of interest is orthogonal to the open contrast used to identify these ROIs for the Experiment 2 analysis, the analysis of Experiments 1 and 3-5 is unquestionably independent of the ROI identification in Experiment 2 (Kriegeskorte et al., 2009).

Using the univariate GLM maps, local maximum $t$-statistic voxels were identified for the group functional maps (Fig. 2 from Experiment 2). Local maxima were identified using a step-up procedure: from an 

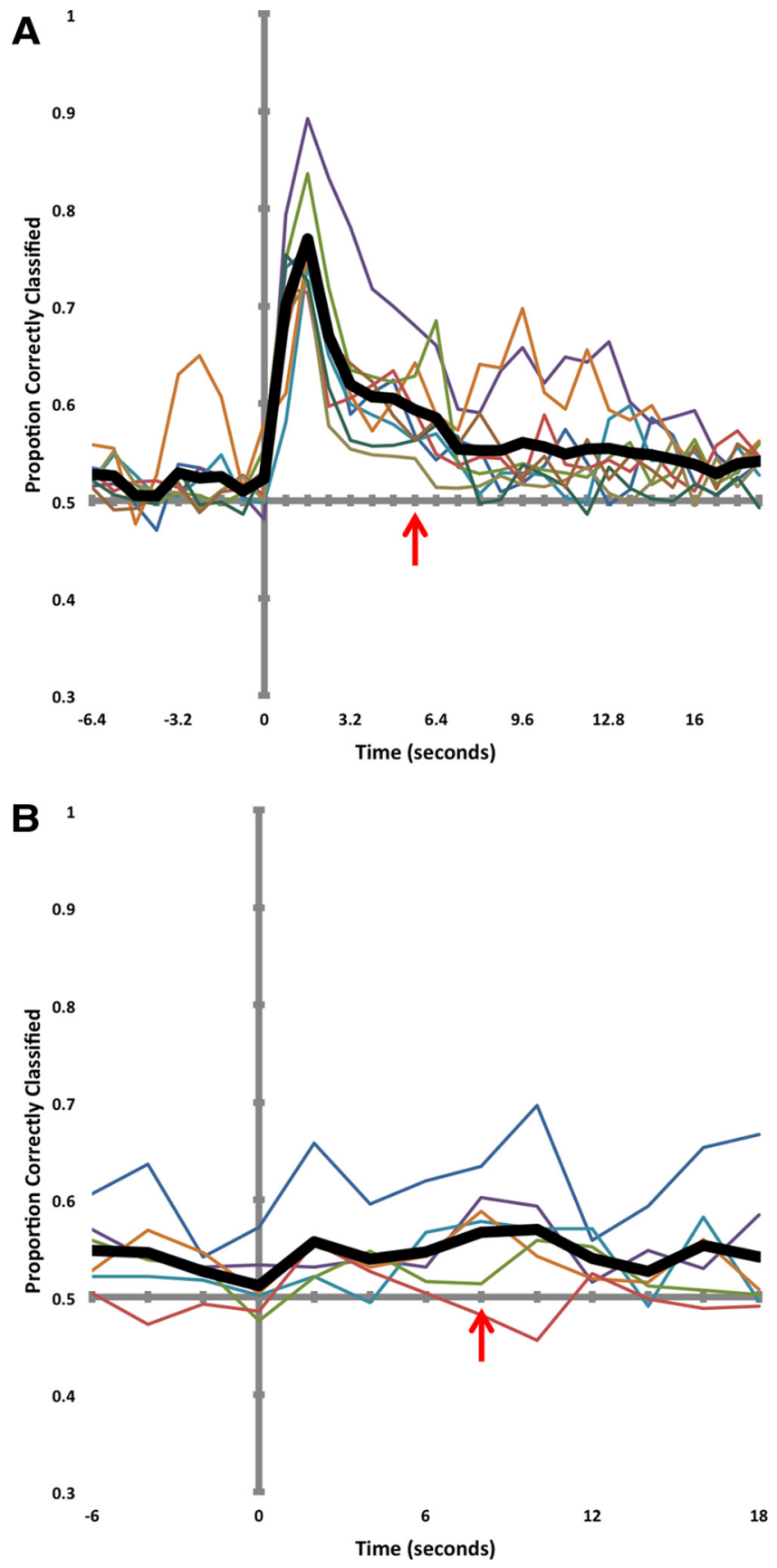

Figure 3. $\quad \boldsymbol{A}, \boldsymbol{B}$, Estimated er-MVPA time courses from lateral ventricles in Experiments $3(\boldsymbol{A})$ and $4(B)$. In this and the following figures, thick black lines represent the mean across subjects and thin colored lines plot single subjects. Red arrows indicate group-based peak TR based on brain regions that showed HRF-like MVPA in Experiments 3 and 4. There is a large initial vocal artifact in Experiment 3, which is most prominent in short-TR acquisitions. There is also lower but sustained time course in both experiments (after initial artifact spike for Experiment 3).

initial threshold of $p<0.05$, we gradually made the threshold more conservative to separate peaks within large contiguous regions. We thus identified local maxima corresponding to brain regions previously associated with RS (e.g., Dux et al., 2006, 2009; Ivanoff et al., 2009; Tombu et al., 2011) and/or general cognition and task performance (e.g., Duncan and Owen, 2000; Duncan, 2001, 2010; Fox et al., 2005). At the same time, we avoided an arbitrary choice of a single threshold that would lead us to consider only one peak within a large contiguous activation containing several foci.

Each local maximum served as the center of an ROI. ROIs were initially drawn as spheres with a radius of 3 (resampled) functional voxels

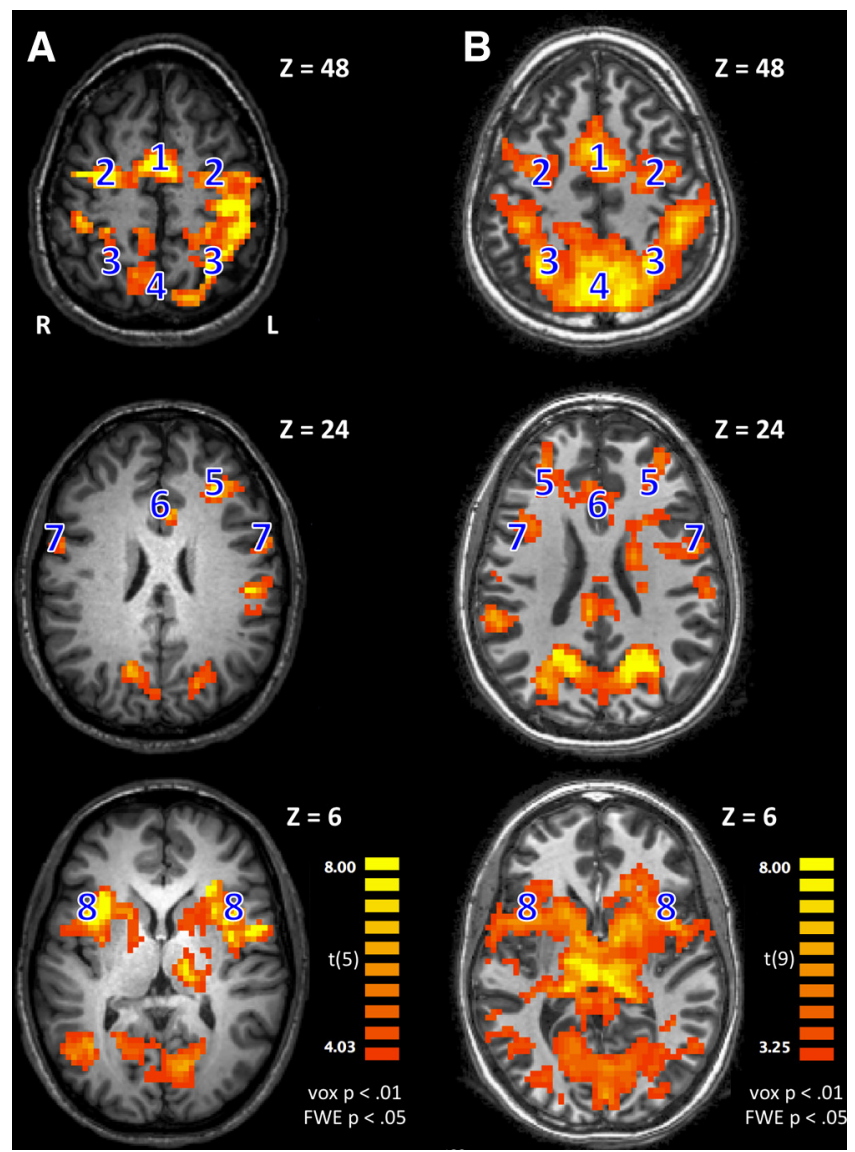

Figure 4. Group random-effects activation maps from Experiments 4 and 5.A, Conjunction of auditory/manual and visual/oculomotor open contrasts in Experiment 4 (correct trials only). $\boldsymbol{B}$, Conjunction of valid RS and RE open contrasts in Experiment 5 . In both experiments, univariate activated regions overlapped with nearly all ROIs defined based upon Experiment 2 . Blue numbers label approximate brain regions relevant for the present analysis: 1, SMA; 2 , FEF; 3, IPS; 4, mSPL; 5, DLPFC; 6, ACC; 7, pLPFC; 8, Al. Each experiment's data are depicted on the brain of a representative subject from that experiment.

(i.e., $9 \mathrm{~mm}$ ). In the course of mapping between functional ( $3 \mathrm{~mm}$ isotropic) and anatomical ( $1 \mathrm{~mm}$ isotropic) coordinate spaces, the ROIs were expanded to include 232 functional voxels comprising a sphere centered on the local maximum (effective radius of $\sim 11 \mathrm{~mm}$ ). In the rare event that this spherical ROI extended outside the acquired fMRI images, the ROI used all acquired voxels, typically $>180$ in each case.

Local maxima for medial structures (e.g., SMA) were shifted to the midline (i.e., their $x$-coordinates took on the value 0 ). This avoided paired bilateral ROIs sampling largely overlapping voxels, and/or single ROIs sampling unequally across hemispheres.

Posterior lateral prefrontal cortex (pLPFC) ROIs for Experiments 1-3 were split such that two spheres were drawn, each centered on a point 9 $\mathrm{mm}$ anterior or posterior to the local maximum. Only the anterior portion of the pLPFC ROI is reported (as posterior lateral prefrontal cortex) to ensure that signal originating in Broca's area related to vocal production did not contaminate MVPA (Ivanoff et al., 2009). Because Experiment 4 did not use vocal responses, the original ROI centered on the local maximum was used. The anterior-shifted ROI did not produce qualitatively different results from the original ROI in Experiment 4.

In addition to the frontal and parietal brain regions analyzed here, local maxima included regions in extrastriate visual cortex, auditory cortex, and motor cortex. These regions are not included in the main results because of their known roles in modality-specific sensory perception or motor production, and because preliminary inspection of MVPA in these regions yielded far above-chance classification consistent with the shape of the BOLD HRF. It is unclear why they survived the conjunction of RS tasks across modalities, although it is possible that peripheral cor- 
Table 3. Results of MVPA for Experiments $1-5^{a}$

\begin{tabular}{|c|c|c|c|c|c|c|c|c|c|c|c|}
\hline \multirow{2}{*}{ Region } & \multirow{2}{*}{\multicolumn{3}{|c|}{ Coordinates }} & \multicolumn{4}{|c|}{$\begin{array}{l}\text { Experiment } 1 \text { (peak }=8 \mathrm{~s} \text {, Bonferroni }=18, \\
N=7 \text { ) }\end{array}$} & \multicolumn{4}{|c|}{$\begin{array}{l}\text { Experiment } 2 \text { (peak }=6 \mathrm{~s}, \text { Bonferroni }=18, \\
N=12)\end{array}$} \\
\hline & & & & \multicolumn{2}{|l|}{ Unsubtracted } & \multicolumn{2}{|l|}{ Artifact subtracted } & \multicolumn{2}{|l|}{ Unsubtracted } & \multicolumn{2}{|l|}{ Artifact subtracted } \\
\hline Left DLPFC & -28 & 40 & 33 & 0.583 & 0.139 & 0.036 & 1.000 & 0.615 & 0.127 & 0.047 & 1.000 \\
\hline Left posterior DLPFC & -40 & 19 & 33 & 0.576 & 0.010 & 0.029 & 1.000 & 0.623 & 0.006 & 0.054 & 0.240 \\
\hline Right DLPFC & 29 & 31 & 33 & 0.570 & 0.005 & 0.023 & 0.083 & 0.594 & 0.006 & 0.026 & 1.000 \\
\hline Left anterior insula & -30 & 10 & 12 & 0.582 & 0.013 & 0.035 & 1.000 & 0.625 & 0.007 & 0.056 & 0.517 \\
\hline Right anterior insula & 32 & 7 & 9 & 0.588 & 0.026 & 0.042 & 1.000 & 0.625 & 0.013 & 0.057 & 1.000 \\
\hline $\mathrm{ACC}$ & 0 & 7 & 38 & 0.634 & 0.035 & 0.087 & 0.102 & 0.686 & 0.000 & 0.117 & 0.016 \\
\hline Posterior ACC & 0 & 4 & 45 & 0.666 & 0.015 & 0.119 & 0.112 & 0.701 & 0.000 & 0.133 & 0.003 \\
\hline SMA & 0 & -8 & 66 & 0.712 & 0.001 & 0.166 & 0.001 & 0.729 & 0.000 & 0.161 & 0.000 \\
\hline Left FEF & -28 & -14 & 48 & 0.733 & 0.000 & 0.186 & 0.002 & 0.805 & 0.000 & 0.237 & 0.000 \\
\hline Medial SPL & 0 & -56 & 48 & 0.626 & 0.011 & 0.079 & 0.044 & 0.647 & 0.000 & 0.079 & 0.324 \\
\hline Left IPS & -25 & -71 & 42 & 0.625 & 0.084 & 0.078 & 0.372 & 0.693 & 0.000 & 0.124 & 0.019 \\
\hline Right IPS & 23 & -68 & 45 & 0.639 & 0.038 & 0.092 & 0.096 & 0.708 & 0.000 & 0.139 & 0.001 \\
\hline Lateral ventricles & & & & 0.546 & 0.027 & & & 0.568 & 0.013 & & \\
\hline
\end{tabular}

${ }^{a}$ For each experiment, each ROI's proportion of correct classification is given before and after artifact subtraction. All $p$ values reflect Bonferroni correction for the number of brain regions examined in the main (RS-defined regions) analysis (see experiment headings). Bolded $p$ values reflect corrected $p \leq 0.05$. Shaded rows indicate ROls consistently exhibiting insensitivity to stimulus and response modality. Parenthetical $y$-coordinates reflect Experiment 4 ROI location (see Materials and Methods). Ventricle coordinates are not given because of variability across experiments as well as variability in shape and location across subjects in Experiments 4 and 5 . Ventricle MVPA and $p$ values reflect MVPA at the time corresponding to the peak response in neural ROls for each experiment (see experiment headings for exact time points). Ventricle $p$ values reflect identical Bonferroni correction to other $p$ values in order to ease comparison of results for artifactual (ventricle) and neural (subtracted) MVPA (Continued).

tices were generally activated in both tasks in anticipation of the onset of a trial. This seems especially plausible given the structure of Experiment 2 (the experiment used for ROI definition) in which the trial types occurred with nonjittered timing in a random order and in which one-third of trials were dual-task and required both modalities of sensory input and motor output. In past work, these regions showed large BOLD amplitude differences across modalities (Ivanoff et al., 2009).

Experiment 5 used a subset of the Experiment 2 ROIs that exhibited failure to decode between sensory and/or motor modalities in Experiments 1-4. These five a priori ROIs included bilateral anterior insula (AI), right dorsolateral prefrontal cortex (DLPFC), and two ROIs in left DLPFC. This subset was selected to evaluate whether failure to decode was the result of a general insensitivity of MVPA in these brain regions or whether it was specific to decoding of sensory/motor modality.

In addition to the ROIs defined with the response selection tasks, we defined MD ROIs (using canonical MD region peak coordinates; Table 2) for a follow-up analysis to ease comparison between the current work and other studies of the MD network. The MD coordinates were drawn from Duncan (2010) and reflect peaks of large regions isolated with a meta-analysis. We placed ROI spheres of identical size to those used in the main analysis at these coordinates. We did not define additional regions based explicitly on the TP network because: (1) the TP and MD networks have been suggested to be equivalent in the prior literature (e.g., Duncan, 2010); (2) the TP network is defined based on resting state functional connectivity rather than task-related activation; and (3) the combination of the MD ROIs with our functionally defined ROIs already covers much of the TP network.

$M V P A$. For all experiments, the entire time course of each voxel in each ROI for each subject was extracted separately from the nonsmoothed 4-dimensional data and $z$-transformed with respect to its own run-level time course. Then, for each trial, the $z$-transformed data from each voxel were entered into an ROI-, subject-, and time point- (locked to the volume acquisition of event onset) specific table labeled with trial (or for Experiment 5, task phase within trial) type (e.g., visual/manual or auditory/vocal) and run number. Thus, MVPA compared auditory-vocal to visual-manual trials for Experiments 1 and 2, auditory-manual to visual-vocal for Experiment 3, auditory-manual to visual-oculomotor for Experiment 4, and manual re- sponse selection to manual response execution for Experiment 5. Data were extracted over the time range -6 to $18 \mathrm{~s}$ (Experiment 3: -6.4 to $18.4 \mathrm{~s}$ ), locked to the event of interest. Data at each time point for each event type were averaged across voxels and then subtracted from each individual voxel's data to remove mean differences between event types that could reflect differences of difficulty or other differences of no interest (Esterman et al., 2009; Tamber-Rosenau et al., 2011).

Independently for each ROI (including canonical MD ROIs; see ROIs), subject, and time point, we performed a leave-one-run-out procedure: all but one run of data were used to train a linear support vector machine that was then tested on the held-out run; this process was iterated until all runs had served as the test data once (3- to 24-fold crossvalidation, depending upon experiment and subject; Table 1). Classifier proportion correct was aggregated to determine an ROI-, subject-, and time point-specific MVPA result. Within an ROI, MVPA results across time points were concatenated to form an ROI- and subject-specific event-related MVPA (er-MVPA) time course (Esterman et al., 2009; Tamber-Rosenau et al., 2011) with chance performance at 0.5 (proportion correct classification) and perfect performance at 1.0.

The set of subject er-MVPA time courses was compared with chance at each time point via paired $t$ test. Chance er-MVPA performance was empirically estimated to rule out artifactual above-chance performance (as a result of, for instance, imperfect balance of number of correct trials of each type per run) by performing 1000 iterations per classifier in which the trial labels were randomized (Esterman et al., 2009; Tamber-Rosenau et al., 2011); typical estimates of chance were well within 0.01 of the 0.5 expected chance level, confirming that nothing about the task design or analysis procedure per se biased chance away from 0.5 . In each experiment, statistical significance was assessed after a Bonferroni correction for the number of ROIs evaluated. No correction was performed for the number of time points assessed within a region because: (1) BOLD-fMRI time points are not independent of adjacent time points, complicating such correction; and (2) when evaluating potential null findings (i.e., absence of modality coding), it is more conservative to adopt a liberal statistical threshold.

Artifact estimation and correction. Initial examination of er-MVPA time courses from Experiments 1-3 revealed in some brain regions an early-onset er-MVPA profile that did not conform to the expected 
Table 3. Results of MVPA for Experiments $1-5^{a}$ (Continued)

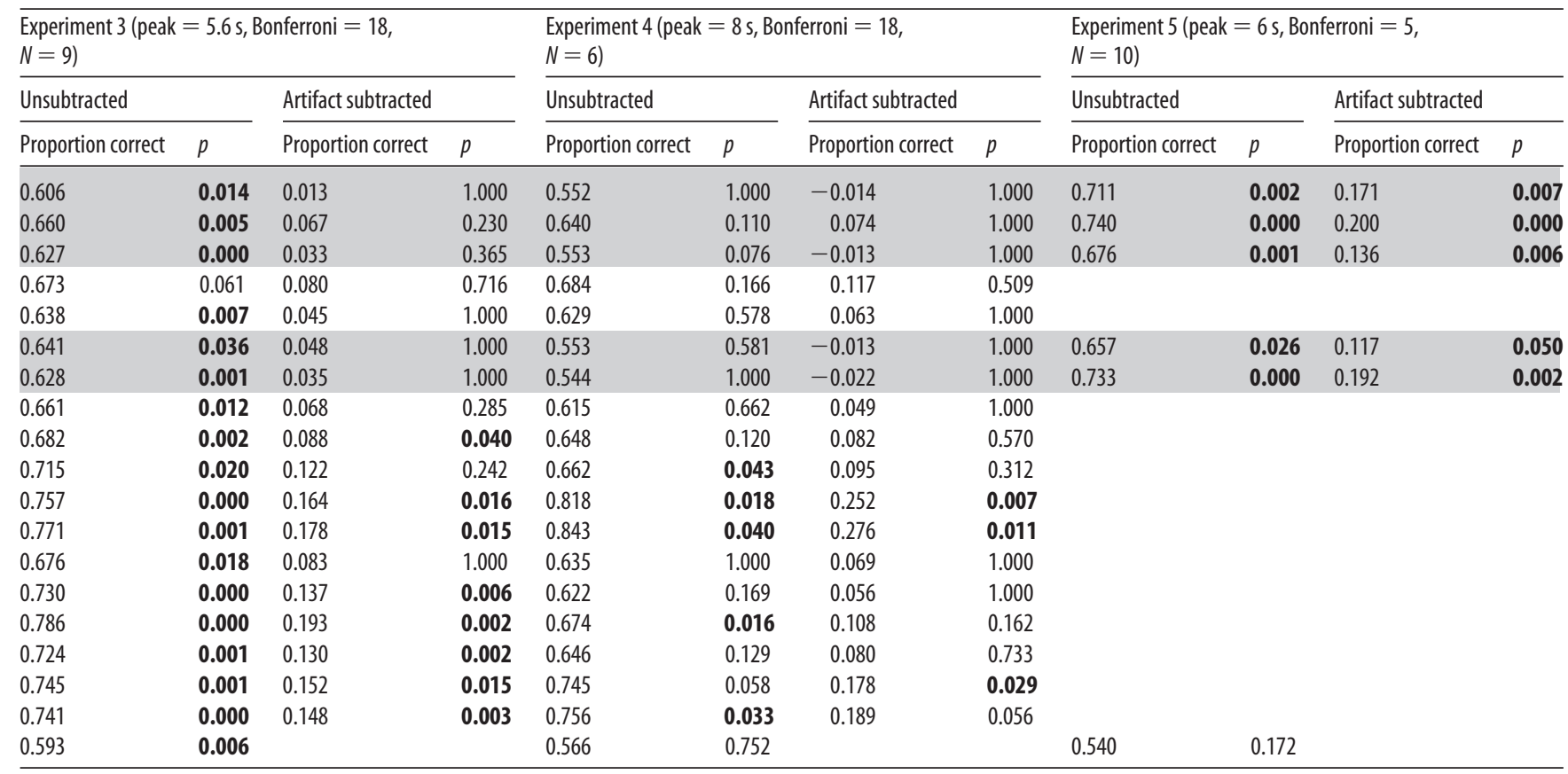

shape of the er-MVPA response (i.e., a canonical BOLD HRF) (see, e.g., Boynton et al., 1996). The unexpected profiles might be the result of artifacts induced by the vocal responses that are made in only one of the two trial types (Dux et al., 2006) in each experiment. Vocal responses are known to induce fMRI artifacts resulting from changes in head position and in the magnetic field (Birn et al., 2004; Huang et al., 2008), and they may also trigger changes in fMRI signal resulting from respiratory fluctuations in chest cavity volume that generate artifacts that are not removable by standard rigid-body motion correction (for a discussion of such respiratory artifacts, see Raj et al., 2000, 2001). Such artifacts were less problematic for the original univariate analyses of Experiments 1-3 because those analyses relied on overlap of activation across modalities and the vocal artifacts were only present in a single modality. By contrast, the new multivariate analyses attempt to detect such differences across modality. To estimate any contribution to er-MVPA of the artifact induced by vocal responses and related motion, ROI spheres were placed identically to those for functionally defined ROIs but centered in the posterior portion of the lateral ventricles, away from any gray matter. Data from these ventricle ROIs were subjected to an identical er-MVPA analysis to the functionally defined ROIs; er-MVPA estimates from four lateral ventricle ROIs were then averaged within-subject to yield a final ventricle ROI erMVPA time course for each subject in each experiment.

Ventricle ROIs showed similar early-onset, non-HRF-like er-MVPA time courses (Fig. 3) to those from the cortical ROIs, suggesting that cortical MVPA results stemmed in part from vocal artifact and related motion. Thus, to correct er-MVPA in cortical ROIs for vocal- and motion-induced artifactual classification, we subtracted, on a subjectby-subject basis, the ventricle er-MVPA time course from each cortical er-MVPA time course, yielding a corrected time course with chance at 0 and perfect MVPA classification at a value of 0.5.

Even though Experiment 4 did not contain any vocal responses, preliminary results showed a similar er-MVPA pattern to those in Experiments 1-3, although without a prominent immediate spike that was presumably evoked by the initiating motion of the vocal response in Experiments 1-3. We reasoned that Experiment 4 could also lead to artifactual MVPA resulting from task-correlated motion, which cannot be fully removed by motioncorrection algorithms used in preprocessing (Power et al., 2012). This was especially a concern in Experiment 4 given that it used a block design that lent itself to preparatory motion, which could have yielded slight head position differences across blocks of trials of different types. Because MVPA relies on stable localization of voxels over event types, preparatory motion could induce artifactual classification.

To estimate any possible artifactual MVPA, we repeated the artifact estimation procedure from Experiments 1-3 with Experiment 4. However, to be certain that our results were not in part driven by sampling subcortical gray matter adjacent to the ventricles, for Experiment 4 we traced the lateral ventricles by hand on the structural MRI images. Starting from the anterior end of the lateral ventricles, individual $1 \mathrm{~mm}$ voxels were marked as being in the lateral ventricle until each lateral ventricle ROI contained an identical volume to that in the spherical ROIs used for all other MVPAs. Thus, separate left- and right-lateral-ventricle ROIs were drawn for each participant. Ventricle voxels were tagged up to the edge of white matter but did not include white or gray matter to ensure that no parenchymal tissue was driving MVPA. In principle, resampling of the ventricle ROIs to the lower-resolution functional coordinate space could have led to the inclusion of functional data from adjacent white matter resulting from partial voluming. However, such white matter spillover would most likely be minimal, and, most importantly, its BOLD signal should not differentiate between modalities.

In all but a single subject in Experiment 4, separate ventricle ROIs were traced for each hemisphere and processed identically to those in Experiments 1-3 to determine er-MVPA time courses. Time courses were then averaged within-subject to attain a single artifact estimated time course for each participant. In the remaining subject, the procedure was identical, except that a single disjoint ventricular ROI was used (with half its constituent voxels from each hemisphere) because the lateral ventricles were too small to allow for separate ROIs in each hemisphere. Ventricle er-MVPA time courses matched sustained low-amplitude non-HRF-like time courses evident in some brain regions. This led us to suspect that a small non-neural contribution to MVPA was being measured in many regions throughout the brain.

To make comparisons between Experiment 5 and Experiments 1-4 on an equal footing, data from Experiment 5 were also subjected to artifact estimation and correction. The procedure was identical to that for Experi- 
ment 4. Once again, a single participant necessitated a combined bilateral lateral ventricle ROI, whereas the remaining participants used paired lateral ventricle ROIs.

ANOVA. Data from Experiments 1-3 consistently showed a pattern of results in which a subset of brain regions exhibited er-MVPA at chance or near-chance levels, whereas other functionally defined ROIs exhibited classification well above chance. To confirm that this pattern would hold in an independent dataset, the regions that consistently failed to classify above chance in Experiments 1-3 were chosen as a priori ROIs for Experiment 4. These regions included bilateral AI and bilateral DLPFC. Thus, we hypothesized that, in a new dataset (Experiment 4), these regions would exhibit significantly reduced MVPA classification compared with the remaining ROIs.

er-MVPA results from all 18 ROIs (based on the Experiment 2 group data, as described in ROIs) from Experiment 4 were entered into an ANOVA with the factors ROI, time point, and subject (treated as a random effect). An interaction contrast was then specified over the time point and ROI factors. Specifically, time points 6 and $8 \mathrm{~s}$ after event onset were contrasted with the remaining time points, and the five a priori regions were contrasted with the remaining 13 regions. To ensure that results were not driven by the selection of time points, additional contrastcontrast interactions were run in which the same or a broader range of time points (4-10 s) were compared either with all remaining time points for which classifiers were calculated (full range, -6 to $18 \mathrm{~s})$, or with a more restricted range $(0-14$ s). Thus, four conceptually identical interaction contrasts were performed.

Meta-analytic Bayesian analysis of evidence for and against MVPA classification. The above analyses each consider a single experiment at a time. It is possible that combining Experiments 1-4 to increase power would change the pattern of results across ROIs, especially given the chronically low power of fMRI experiments of typical sample size (see, e.g., Yarkoni, 2009). Thus, results from Experiments 1-4 were pooled via a meta-analytic Bayesian $t$ test approach (Rouder et al., 2009; Rouder and Morey, 2011). To follow up on the results from the functionally defined ROIs and ease comparisons to other work examining the MD network, this analysis was also repeated for the canonical MD ROIs (see ROIs).

The Bayesian $t$ test (Rouder et al., 2009) calculates, for a "default" noninformative prior, the Bayes factor: the ratio of evidence in favor of the null hypothesis (i.e., "the region classifies at chance levels") to that in favor of the alternative hypothesis (i.e., "the region does not classify at chance levels"). We take the inverse of this ratio so that large numbers indicate substantial evidence for decoding and small numbers indicate substantial evidence for failure to decode (i.e., evidence in favor of the null hypothesis) (Rouder and Morey, 2011). A value of 1 would indicate equal evidence in favor of and against the null.

The meta-analytic extension of the Bayesian $t$ test approach (Rouder and Morey, 2011) allows integration of multiple experiments into a single Bayesian $t$ test. Evidence ratios from multiple ROIs (in either the single-experiment or meta-analytic tests) then are directly comparable to one another. Thus, to compare Experiment 5 with Experiments 1-4, we
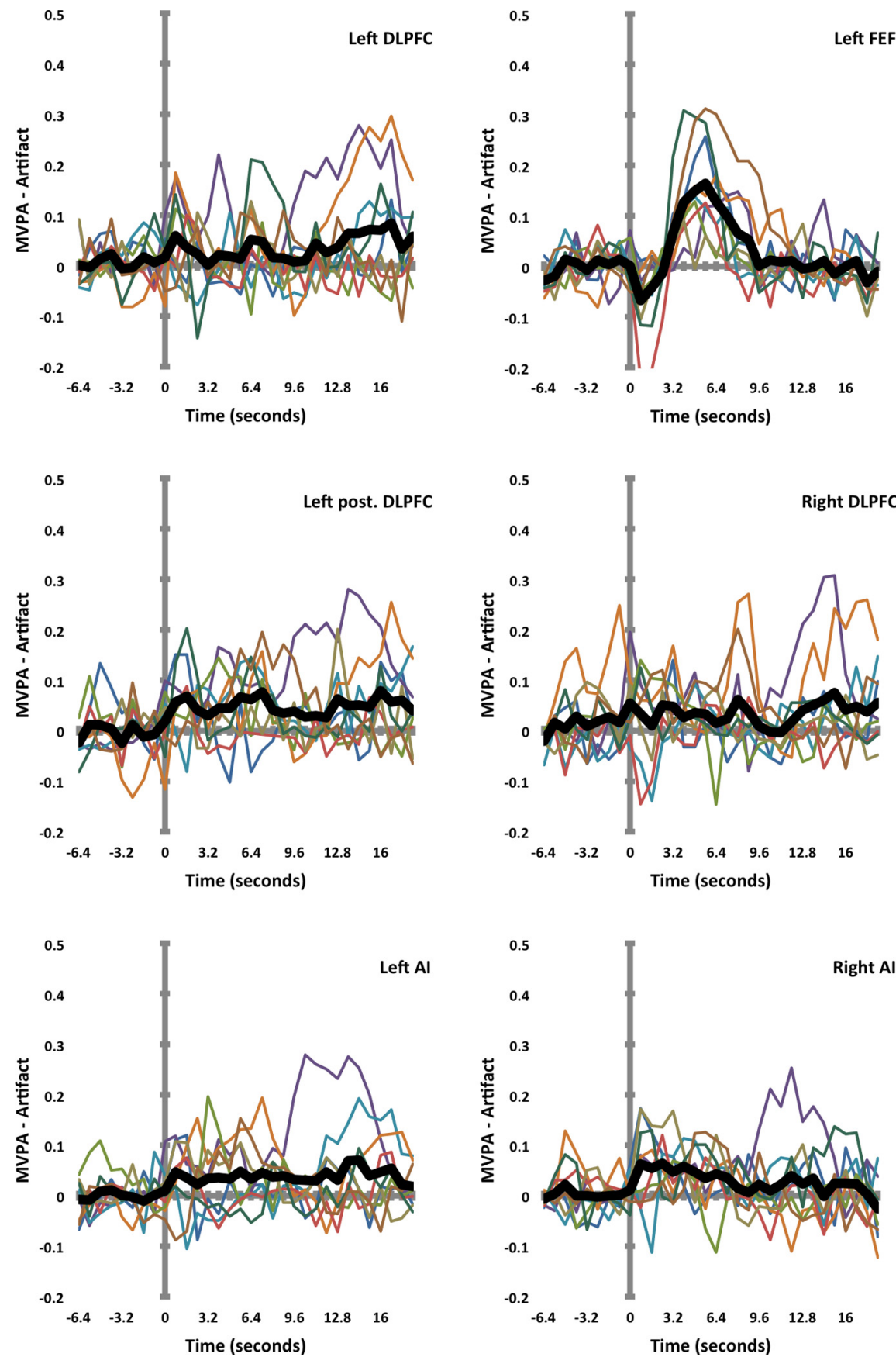

Figure 5. Event-related MVPA time courses from selected brain regions in Experiment 3 , after subtraction of artifact estimates from each subject's time course (compare with Fig. $3 A$ ).

also calculated the single-experiment Bayesian $t$ test (Rouder et al., 2009) for Experiment 5.

All Bayesian tests were performed using R code implementing the test of Rouder and Morey (2011) and kindly provided by Jeffrey N. Rouder (personal communication, January 16, 2012).

\section{Results}

Univariate GLMs of sensorimotor activation overlap

Univariate GLM results for Experiments 1-3, described in detail in their respective original publications (Dux et al., 2006, 2009; Tombu et al., 2011), included conjunction activations across task modalities in several lateral PFC regions, AI, medial frontal cortex, premotor cortex near the FEF, and intraparietal sulcus (IPS)/ superior parietal regions. Figure 2 illustrates the univariate GLM 

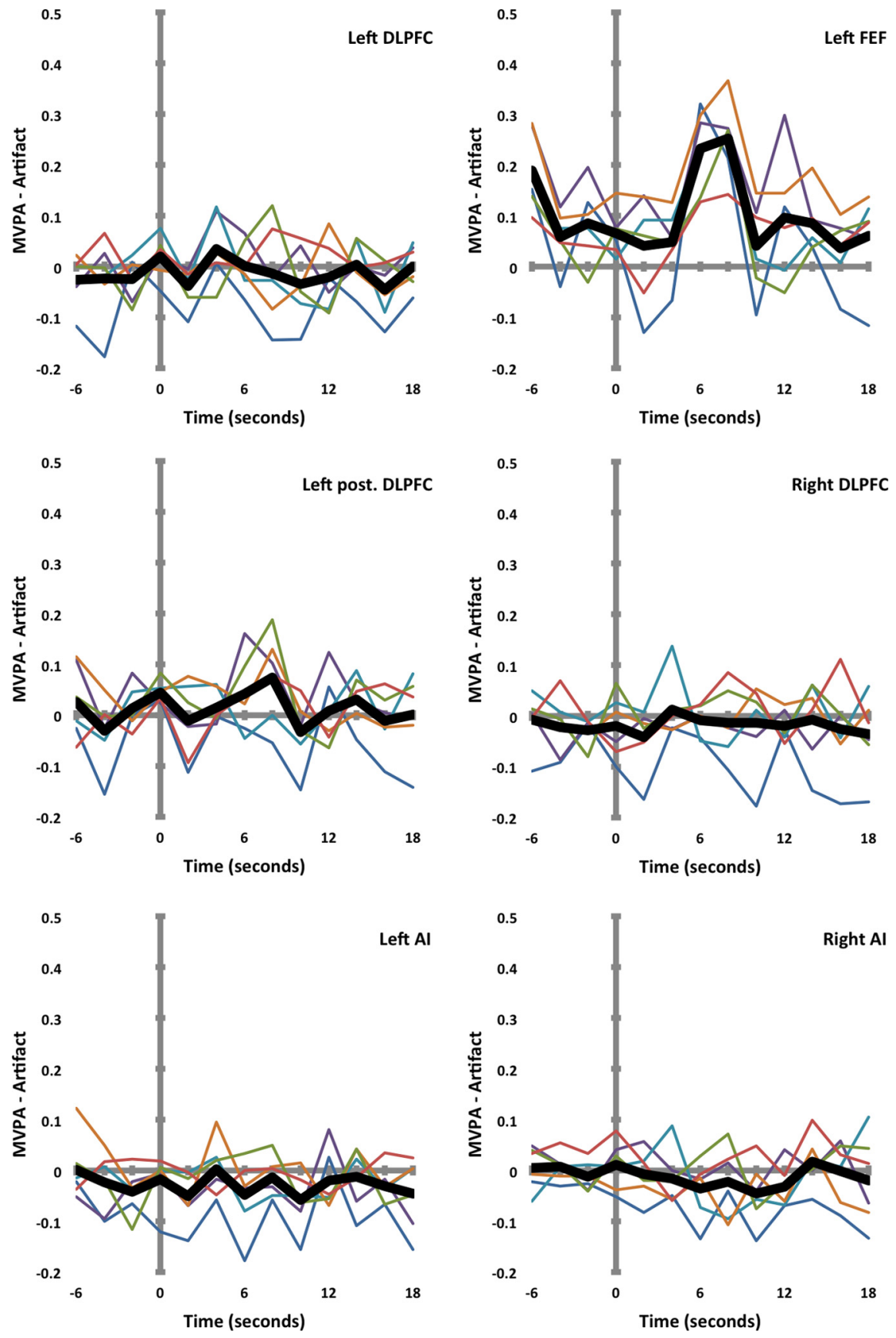

Figure 6. Event-related MVPA time courses from selected brain regions in Experiment 4, after subtraction of artifact estimates from each subject's time course (Fig. 3B).

from Experiment 2 on which the ROIs for all experiments were based. Experiment 4 yielded univariate activations in several regions across the brain for the conjunction of open contrasts of auditory/manual and visual/oculomotor RS (Fig. 4A), and Experiment 5 also yielded extensive activations for the conjunction of the open contrasts of RS and RE (Fig. 4B). In each experiment, a large set of frontal and parietal brain regions broadly consistent with the TP/MD networks was activated by the conjunction contrasts (Table 2). Thus, these experiments are suitable for comparing tasks across modalities within the TP/MD networks and other frontal and parietal regions.

Two aspects of the univariate analyses are worth noting. First, although the functionally defined regions of the main analysis substantially overlap the large MD and TP regions reported pre- viously, the peaks of these functionally defined ROIs are displaced from canonical peak coordinates of MD regions derived from a meta-analysis (Duncan, 2010) (Tables 2 and 3). This displacement could be the result of random variability across different subject cohorts, but it also could reflect task differences (i.e., the regions we report could be specialized for RS tasks). Second, the lateral prefrontal (and parietal) activations evident in these univariate analyses obviate a potential concern about the present work: Because our paradigms placed little demand on working memory (WM), and because the parietal and prefrontal cortex are often linked to WM processes (e.g., Levy and GoldmanRakic, 2000; Sala et al., 2003; Courtney, 2004; Todd and Marois, 2004; 2005; Nee et al., 2012), it could be argued that our RS tasks did not provide sufficient sensitivity to detect modality-specific effects that WM tasks would. However, the univariate results show that the DLPFC, pLPFC/IFJ (inferior frontal junction), and other frontoparietal regions were robustly activated with the RS tasks, even under limited WM demands. Furthermore, WM demand is not sufficient for LPFC coding of stimulus information when measured via MVPA (Christophel et al., 2012; Riggall and Postle, 2012). Thus, there is little reason to believe that WM tasks would have been preferable to RS tasks for investigating the modality specificity of prefrontal and parietal regions.

\section{MVPA of modality}

In the experiments manipulating modalities across RS tasks (Experiments 1-4), DLPFC and AI consistently exhibited erMVPA classification that was of very low magnitude and indistinguishable from chance. In contrast to these results, the remaining regions (e.g., FEF) all exhibited significantly above-chance er-MVPA classification in at least one, and generally in several, experiments (for full results of the MVPA, see Figs. 5 and 6; Table 3). In each experiment, AI and DLPFC yielded non-HRF-like, flat er-MVPA time courses that are inconsistent with these regions coding the modalities of task stimulus or response, whereas other brain regions, such as the FEF, yielded HRF-like er-MVPA time courses consistent with coding of modality. Finally, for at least one region that has been previously implicated in amodal RS mechanisms (e.g., Dux et al., 2009), the left pLPFC/IFJ, the evidence for modality coding is somewhat equivocal, as we have found above-chance decoding in this region in only two of the four RS experiments (Experiments 1 and 2). However, in this left pLPFC region (and unlike in AI and DLPFC), er-MVPA time courses in Experiments 1-4 appear to reflect an HRF-like time course with a peak at $\sim 6-8 \mathrm{~s}$ after trial onset (Fig. 7). This finding suggests the possibility that failure to find statistically significant decoding in some experiments in left pLPFC may be the 
result of low power; we address this possibility below using a meta-analytic technique. Nevertheless, the consistent results in AI and DLPFC across Experiments 1-4 provide the first two pillars (replication of the null and a demonstration of sensitivity to find significant coding in other brain regions) upon which we base our conclusion that null results in these brain regions reflect a correct rejection rather than a miss of true coding of modality.

\section{ANOVA: do AI and DLPFC contain less information about modality than other regions of overlap?}

To confirm our conclusion from Experiments $1-3$ that AI and DLPFC were likely amodal (jointly with respect to sensory and motor modality, which are confounded in these experiments), we explicitly compared modality coding in AI and DLPFC versus the remaining ROIs in an independent dataset, Experiment 4, the auditory-manual/visual-oculomotor RS experiment. This experiment removes any possibility of false-positive decoding resulting from vocal artifact because no vocal responses were made. In addition, by using the a priori ROI localization and, furthermore, by testing the a priori categorization (based on Experiments 1-3) of our ROIs as amodal (AI, DLPFC) versus modality-sensitive (the remaining ROIs), we ensure that we do not "double-dip" by generating and testing hypotheses on the same dataset. ANOVA on MVPA results from Experiment 4 revealed main effects of ROI $\left(F_{(17,85)}=11.63, p=1.16 \times 10^{-15}\right)$ and time point $\left(F_{(12,60)}=\right.$ $\left.5.48, p=3.38 \times 10^{-6}\right)$ as well as an ROI $\times$ time point interaction $\left(F_{(204,1020)}=2.46, p=3.03 \times 10^{-20}\right)$. The main interaction contrast (interaction of time points $6-8$ vs all remaining time points, AI and DLPFC vs all remaining ROIs) was significant $\left(F_{(1,1020)}=115.28, p=1.49 \times 10^{-25}\right)$. This result indicates that the five a priori ROIs (bilateral AI and DLPFC) contained significantly less information about the sensory and/or motor modality than did the remaining 13 ROIs, as assessed by event-related MVPA.

Alternative interaction contrasts within the time point factor (time points $4-10$ vs all remaining, time points $6-8$ vs remaining of $0-14$, time points $4-10$ vs remaining of $0-14$ ) yielded similar results $\left(F_{(1,1020)}\right.$ of $41.03,117.63$, and 43.16 , yielding $p$ values of $2.29 \times 10^{-10}, 5.16 \times 10^{-26}$, and $8.02 \times 10^{-11}$, respectively), thereby ensuring that any significant results were not the result of selection of the exact time points chosen.

\section{Bayesian $\boldsymbol{t}$ tests and meta-analysis of modality sensitivity}

The MVPA results represent a null effect in AI and DLPFC. Although this null effect was consistent across four experiments and was obtained in the context of positive effects in other ROIs, we sought further evidence that DLPFC and AI do not code modality, especially in light of the equivocal results in left pLPFC. To assess evidence in favor of amodality, we turned to a Bayesian technique that can assess the degree of evidence in favor of the null hypothesis as well as against the null hypothesis (Wetzels et
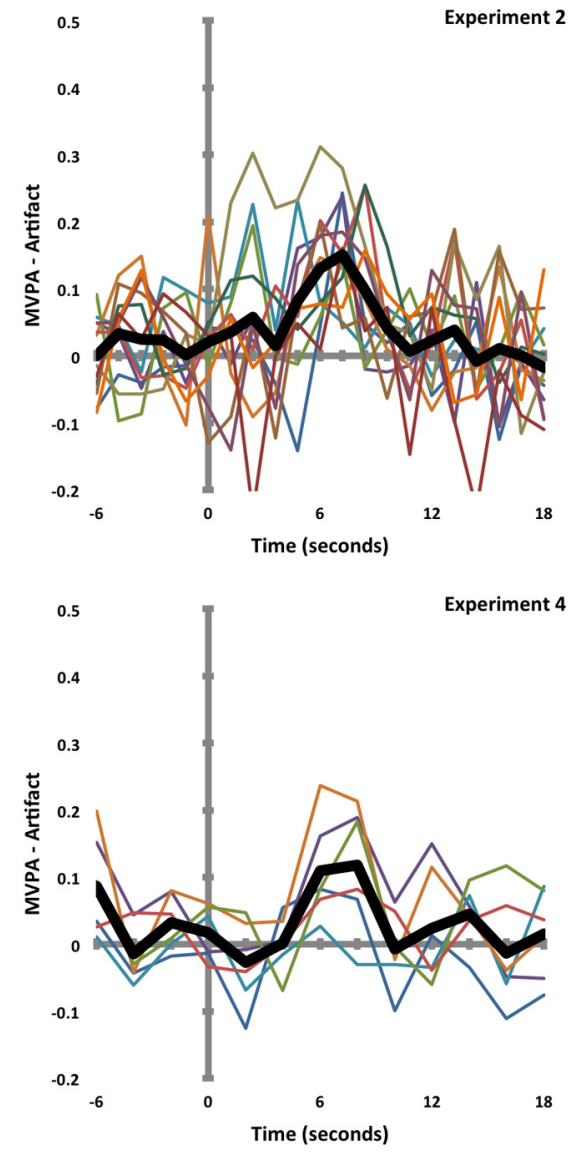

Experiment 1

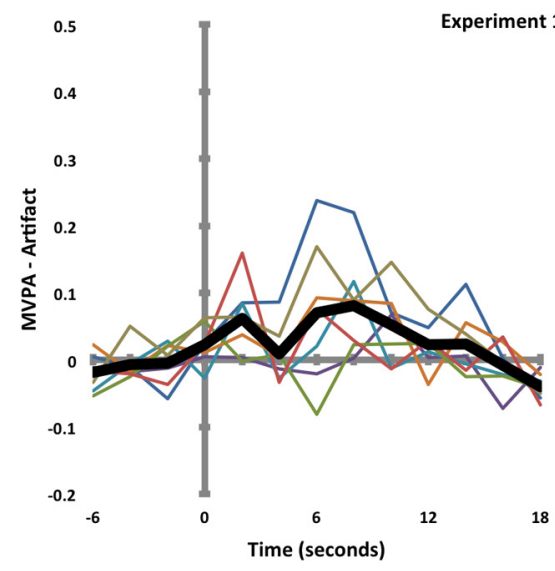

Experiment 3

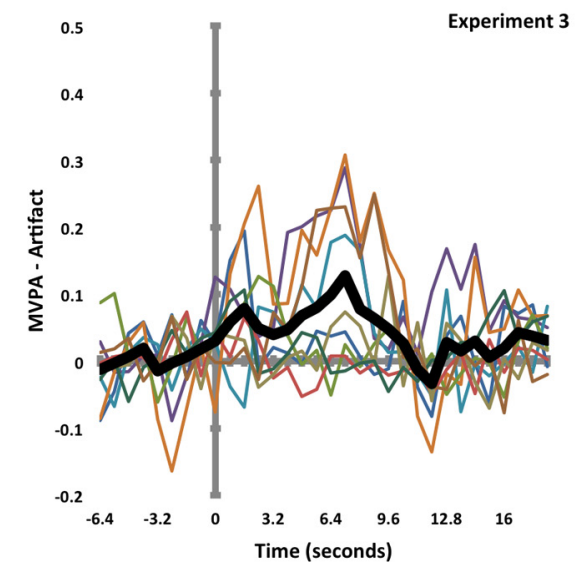

Figure 7. Event-related MVPA time courses from left pLPFC in Experiments 1-4. Although the er-MVPA time courses in each experiment show an HRF-like shape, the individual experiment peaks only rise to significance in Experiments 1 and 2 (Table 3).

al., 2011). We conducted a Bayesian meta-analytic $t$ test (Rouder and Morey, 2011) on data from Experiments 1-4, with a total of 34 subjects, to calculate the Bayes factor. We then inverted this Bayes factor such that it may be read as, "the data are [Bayes factor] times more likely to have occurred under the alternative hypothesis than under the null hypothesis" (Wetzels et al., 2011), with more extreme Bayes factors interpretable as stronger evidence. Figure 8 plots log-transformed inverted Bayes factors such that numbers far $>1$ indicate strong evidence that the ROI can distinguish between perceptual and/or motor modalities and numbers far $<1$ indicate strong evidence that the ROI does not distinguish between perceptual and/or motor modalities.

As Figure 8 makes clear, evidence for successful classification of modality is extremely strong for the 13 ROIs we do not hypothesize to be amodal (plotted in blue). However, the five amodal ROIs, plotted in red, are weakly (left AI, inverted Bayes factor = 5.05; right DLPFC, inverted Bayes factor $=7.97$ ) or negligibly (right AI, left DLPFC; inverted Bayes factors range 0.68-2.10) sensitive to perceptual or motor modality of RS, except for left posterior DLPFC, which did encode modality (Fig. 8, inset, which plots non-logarithm-transformed inverted Bayes factors). Evidence for modality selectivity in the most modality-selective (highest inverted Bayes factor) of the four remaining amodal regions, right DLPFC, remains approximately three orders of magnitude weaker than in the next weakest region, right FEF. In left pLPFC, where single-experiment MVPA results were more equivocal (Fig. 7; Table 3), the meta-analysis yielded an inverted Bayes factor of $\sim 10^{5}$, suggesting that left pLPFC does encode modality. These meta-analytic results provide the third pillar in 


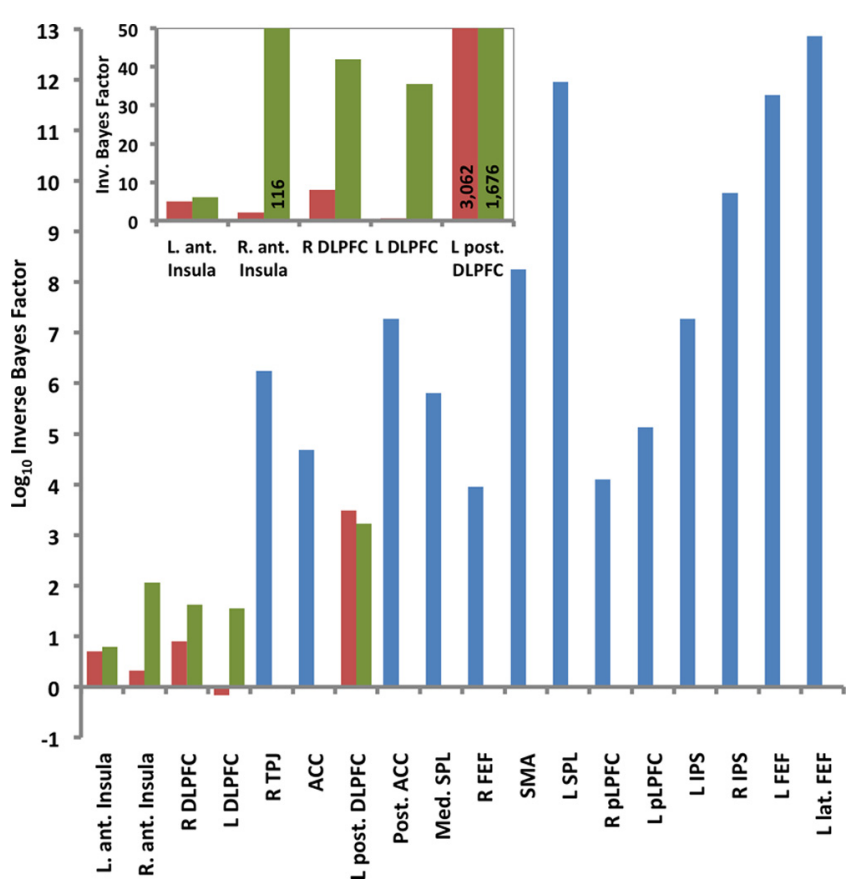

Figure 8. Bayesian analysis of modality coding pooled across Experiments $1-4$ and process coding in Experiment 5. Main plot, The log (base 10) transformed inverted Bayes factor (i.e., larger numbers represent evidence for classification) is plotted for each brain region. Red bars represent Experiments 1-4 meta-analysis for brain regions that appear to be insensitive to modality, based upon examination of MVPA time courses in Experiments 1-4 and the ANOVA in Experiment 4. Blue bars represent Experiments 1-4, remaining brain regions. Green bars represent Experiment 5 (process). Inset, Inverted Bayes factors are plotted (without the log transform) for putative modality-independent regions. Colors are as in main plot. Numbers overlaid on bars denote values exceeding the scale of the plot.

support of our conclusion that null results in AI and DLPFC reflect a true absence of coding.

\section{MVPA and Bayesian $t$ test of cognitive process}

Experiments 1-4 compared modalities and resulted in a consistent null effect of modality decoding in AI and DLPFC. Although these results suggest that these brain regions are not sensitive to task modalities, there are at least two other possible interpretations. First, it is conceivable that hemodynamic or anatomical properties of AI and DLPFC make MVPA methods relatively insensitive in these regions. Second, the method's sensitivity may be sufficient, but these brain regions may not encode any specific task dimensions at all, making a failure to decode modality in particular less surprising. To address these issues, Experiment 5 compared two tasks that differed in process (RS vs RE) but not in modality. Thus, this experiment allowed us to determine whether our failures of decoding in the previous experiments were specific to modality or whether these null results would generalize to other task dimensions.

Application of MVPA to the a priori ROIs in DLPFC and AI defined in Experiment 2 and determined to be amodal in Experiments 1-4 yielded above-chance er-MVPA decoding of task process (Fig. 9). This result is in stark contrast to decoding of task modality, which was consistently at chance across Experiments $1-4$. Thus, failure to classify modality above chance in these regions in Experiments 1-4 is not driven by limitations in the sensitivity of our methodology, or of fMRI or MVPA in general, in AI or DLPFC. This result provides the fourth pillar supporting our conclusion that null results in AI and DLPFC reflect a true absence of coding: we have demonstrated coding of another task dimension in these regions. This result also constrains accounts of the functional roles of AI and DLPFC: they do not encode information that is abstracted of all task dimensions, as one might expect from a completely general-purpose resource (e.g., Norman and Bobrow, 1975) or unified executive process (e.g., Baddeley and Hitch, 1974). Instead, the same regions that fail to code modality do code other task dimensions, namely, process.

The Bayes factors for AI and DLPFC were also calculated in Experiment 5 to assess, in a manner directly comparable with the Bayesian meta-analysis of Experiments 1-4, whether these regions classify RS from RE (Fig. 8). In left posterior DLPFC, where modality is strongly coded, process is strongly coded as well. Experiment 5 Bayes factors for the four amodal regions (bilateral $\mathrm{AI}$, bilateral DLPFC) ranged from slightly greater (in left AI) to approximately two orders of magnitude greater than the Experiment 1-4 meta-analytic Bayes factors for the other regions, despite drawing from a pool of subjects less than one-third the size of that used for Experiments 1-4. Thus, these regions are sensitive to task process (with the possible exception of left AI), providing additional evidence that, if AI and DLPFC were sensitive to modality, our analyses should have demonstrated that sensitivity.

\section{Canonical MD network}

To facilitate comparisons between the present findings and the broader MD literature, we repeated the MVPA and Bayesian test procedures on all five experiments for the set of peak MD regional coordinates reported by Duncan (2010). This analysis supplements rather than replaces analysis of the functionally defined ROIs because neither these canonical MD coordinates nor the functional (RS-defined) ROIs represent the totality of the MD network, which includes large swaths of cortex (e.g., Duncan and Owen, 2000); these larger regions are not suitable for the present analyses comparing MVPA across ROIs because they vary substantially in size across regions, which would lead to potential differences in power across regions. The MD regions were defined based on a meta-analysis of neuroimaging studies of a wide range of behavioral tasks that did not specifically manipulate response selection (Duncan and Owen, 2000; Duncan, 2010). As a result, although the functionally defined RS ROIs are within or adjacent to the canonical MD regions, there are discrepancies between the center coordinates of these ROIs and the canonical MD ROIs (Table 2).

In the canonical MD regions, we observed a pattern of results in which some regions strongly code modality whereas others do not (Fig. 10). This is similar to the findings in the RS regions and suggests that, even among the canonical MD regions, there is heterogeneity of modality representation. However, the specific areas that appear to be amodal from this analysis are only partially consistent with those isolated with the functionally defined RS ROIs (compare Fig. 10 with Fig. 8). Specifically, using the canonical MD regions (and following the naming convention of Duncan, 2010, but with the addition of the prefix "c," for "canonical," to avoid confusion with RS ROIs), right anterior insula/frontal operculum (right cAI/FO), left rostrolateral PFC (left cRPFC), anterior cingulate cortex, and presupplementary motor area (cPreSMA) do not appear to encode modality. Of these amodal regions, right cAI/FO weakly encodes cognitive process (Bayes factor of 4.3), whereas cPreSMA strongly encodes cognitive process (Bayes factor of 162.6). Thus, the clearest replication between the RS and canonical MD ROIs was in right $\mathrm{AI}$ and right cAI/FO (Table 2). The correspondence of other ROIs is somewhat less clear (e.g., compare RS DLPFC with cRPFC and cIFS), although together the canonical MD and RS ROIs seem to tile the 
overall spatial regions reported in the original meta-analysis of $\mathrm{MD}$ regions (Duncan and Owen, 2000). As a whole, these results indicate that most regions in both the response-selection-specific and broadly construed MD networks code modality, but a smaller subset of regions (all in prefrontal cortex and medial frontal cortex) is amodal.

\section{Discussion}

Our main conclusion (that AI and DLPFC are insensitive to modality) is meaningful because: (1) these regions exhibit amodality across four pooled experiments; (2) process coding in AI and DLPFC and modality coding in other regions suggest sufficient sensitivity to detect modalityspecific processing had it existed in $\mathrm{AI}$ and DLPFC; and (3) even if amodality is too strong a claim, these regions are significantly less modality-sensitive than other regions. Amodality in functionally defined AI and DLPFC and at canonical MD coordinates for additional regions bolsters our general view that the RS and MD networks do not function as monolithic units. Instead, subsets of these networks in prefrontal cortex are candidate loci for central amodal processing.

This study also has methodological ramifications: numerically low but significant decoding may be explained by nonneural phenomena. Because MVPA does not average across space, it may be particularly susceptible to motion and other artifacts, especially those correlated in time with task performance. Some artifacts persist past the volume of motion and cannot be removed through realignment or regression (Power et al., 2012). Thus, we suggest future studies estimate and control for artifactual sources of decoding.

Prior studies posited that frontoparietal regions (depending on the study, DLPFC, pLPFC/IFJ, dorsal PM/FEF, IFG, $\mathrm{AI}$, medial frontal structures, or IPS/SPL) have characteristics of an amodal bottleneck (Dux et al., 2006, 2009; Ivanoff et al., 2009; Tombu et al., 2011), in part because of spatial overlap of RS-evoked fMRI activations across modalities. Other work demonstrated frontal or parietal overlap for attention shifts between sensory modalities (Shomstein and Yantis, 2004) or for visual and haptic spatial selectivity (Sathian et al., 2011). Finally, resting state functional connectivity (Fox et al., 2005) and neuroimaging meta-analyses (Duncan and Owen, 2000; Duncan, 2001, 2010; Niendam et al., 2012) indicated broad frontoparietal recruitment across tasks and modalities. However, these univariate studies were insensitive to finer-grained segregation of modality-sensitive neural ensembles within macroscopic brain regions. MVPA is sensitive to such patterns (Kamitani and Tong, 2005; Kamitani and Sawahata, 2010; Op de Beeck, 2010a,b). To our knowledge, we present
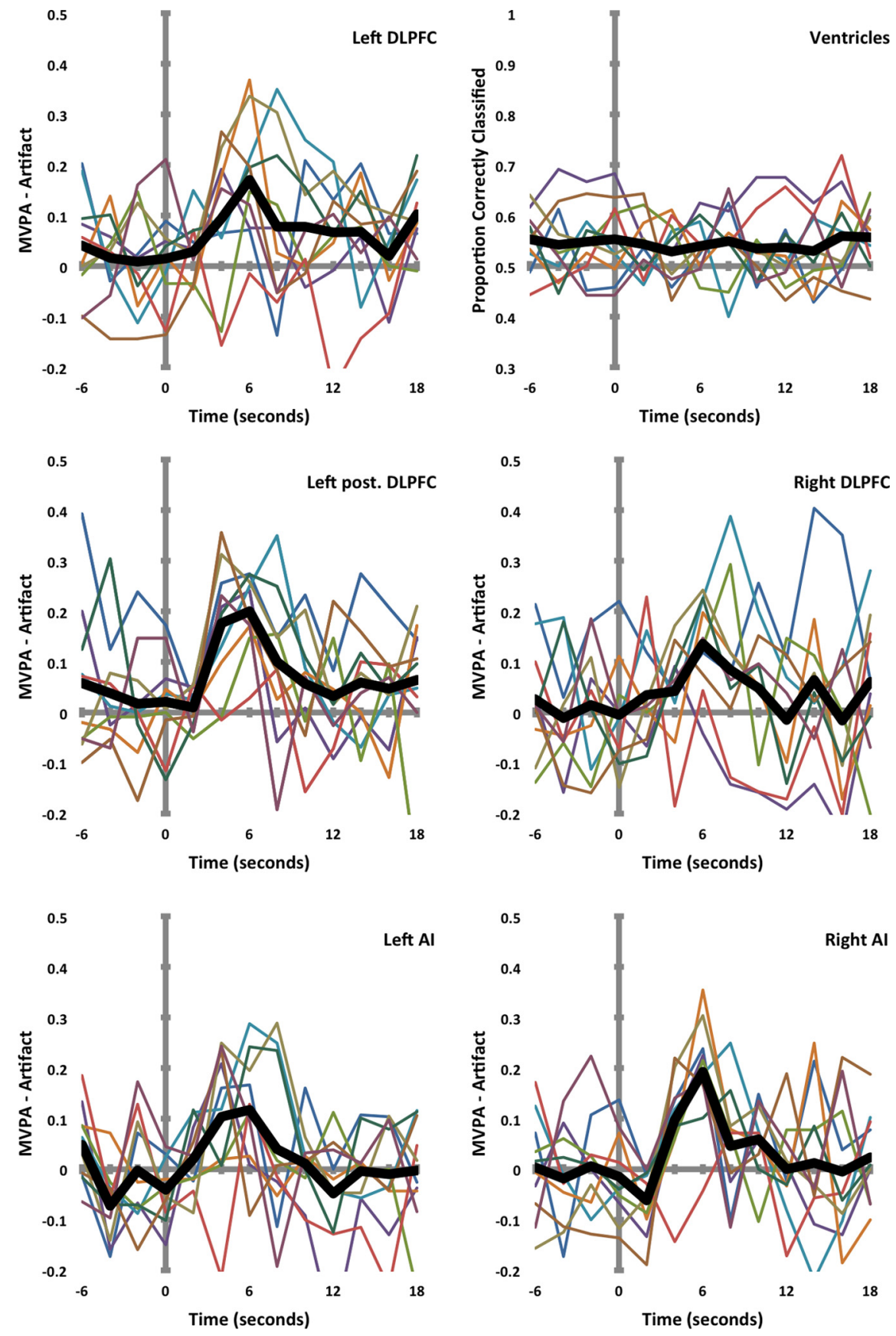

Figure 9. Artifact-subtracted event-related MVPA time courses in the five a priori regions of Experiment 5, as well as ventricle er-MVPA time course (i.e., artifact time course) without any subtraction. Although none of these regions coded modality (see Experiments 1-4), each nonventricle region codes process in Experiment 5.

the first MVPA of modality sensitivity throughout frontoparietal cortex (for application in a restricted region, see Dux et al., 2009). In contrast to univariate studies, we find that most frontoparietal regions code modality. Only two RS-defined structures (AI and DLPFC) consistently failed to code modality; a corresponding right $\mathrm{AI}$ region at canonical coordinates also failed to decode modality (as did a few additional canonical ROIs). These are strong candidates for further study as loci of central bottleneck processes that instantiate amodal capacity limits on information processing (Dux et al., 2006, 2009; Ivanoff et al., 2009; Tombu et al., 2011), although other criteria for a central bottleneck than amodality need also be met by these regions. Such processes could be equivalent to a "router," linking information to re- 


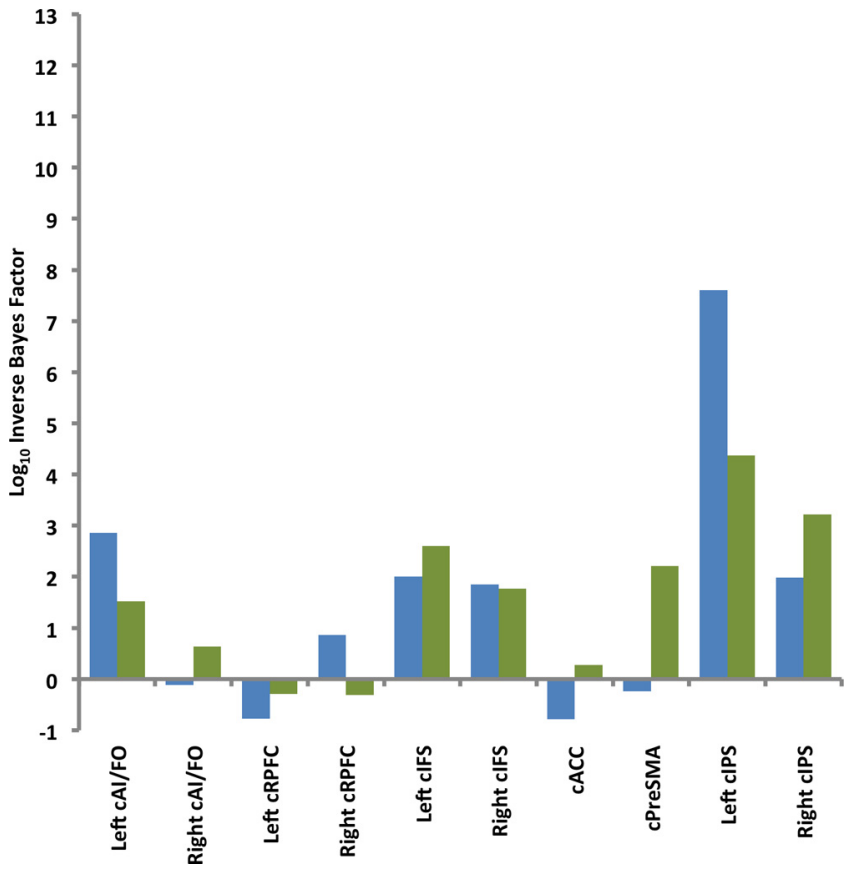

Figure 10. Bayesian analysis of modality coding pooled across Experiments 1-4 and process coding in Experiment 5 for ROls derived from canonical MD region coordinates (Duncan, 2010). The log (base 10) transformed inverted Bayes factor (i.e., larger numbers represent evidence for classification) is plotted for each brain region. Blue bars represent Bayesian metaanalysis of Experiments 1-4 (modality). Green bars represent Experiment 5 (process).

sponses based on task guidelines without regard for modality (e.g., Zylberberg et al., 2010). A bottleneck in AI is particularly supported by prior research: using a dual-task paradigm, Tombu et al. (2011) demonstrated delay of AI peak activation for highload trials at short stimulus onset asynchronies (load $\times$ stimulus onset asynchrony interaction), matching predictions for a serial bottleneck. Our complementary finding that regions (e.g., FEF, IPS, RS-defined medial frontal regions) exhibiting overlap of activation across modalities nevertheless code modality when assessed with MVPA sounds a cautionary note for research concluding amodal processing from overlap alone (e.g., Cowan et al., 2011): if MVPA can decode in these regions, then at least some neurons within them are likely sensitive to modality.

Primate electrophysiology demonstrates both modalityspecific and multimodal neurons within the same region of prefrontal cortex. Wollberg and Sela (1980), Watanabe (1992), and Artchakov et al. (2007) described neurons sensitive to both vision and audition, whereas Keysers et al. (2003) found insensitivity to sensory modality and motor performance in ventral premotor cortex audiovisual mirror neurons. Interspersed with these multimodal cells, however, were modality-specific cells, casting doubt on the existence of brain regions exclusively populated by amodal cells (at least within the species and regions examined). However, even if some cells in AI and DLPFC are modalityspecific, inhibitory connections between such neurons could suppress simultaneous activity for multiple modalities, which would effectively lead to signaling one modality at a time (similar to biased competition between stimuli) (Desimone and Duncan, 1995), consistent with an amodal functional bottleneck. In any case, AI and DLPFC are significantly less modality-specific, at least in the context of RS tasks, than other activation-overlap ROIs (see Experiment 4 ANOVA). There is likely a gradient (Table 3; Fig. 8) across TP/MD regions, from very (e.g., FEF) to minimally (AI and DLPFC) modality-sensitive.
Both fMRI (Cole et al., 2011; Reverberi et al., 2011; Woolgar et al., 2011a,b) and electrophysiology (Rainer et al., 1998; Asaad et al., 2000; Warden and Miller, 2007; Meyers et al., 2008; Cromer et al., 2010; Roy et al., 2010; Warden and Miller, 2010) have demonstrated LPFC encoding of task properties other than modality, including stimulus identity, motor responses, and stimulus-response mapping rules, although others have found little or no LPFC stimulus encoding (Bode and Haynes, 2009, supplemental Analysis III; Christophel et al., 2012; Riggall and Postle, 2012). One might suppose that regions encoding such task properties would also encode modality because modality may be essential to stimulus identity or motor response. This supposition requires reconciliation between our findings (that some LPFC regions do not code modality) and these previous findings. Setting aside the possibility that LPFC coding for task properties is spatially segregated from the amodal regions identified here, one possible reconciliation is that LPFC does not encode all features of stimulus, response, or rule but instead encodes only necessary task-relevant information to distinguish between contextually relevant categories of stimulus, response, or rule. Previous findings that LPFC encodes such information as stimulus identity might not reflect absolute coding of all features (e.g., "mediumdark roasted Sumatran coffee brewed in a French press, poured into a 20-ounce mug"), but instead reflect context-specific coding of one exemplar (e.g., "coffee") versus a few alternatives (e.g., "water," "tea," "juice") relevant for a specific decision (beverage choice). In this framework, if stimulus modality (hearing vs seeing "coffee") or response (uttering "coffee" vs pointing to the coffee) were unimportant in the task context, they would be abstracted in favor of task-relevant information. It is conceivable that, under some conditions, modality could be the task-critical information to be encoded, but because modality was confounded with specific stimulus identity in the present tasks, we would not expect LPFC to encode modality here. Future work will need to directly test this hypothesis and also clarify whether the observed modality coding in some LPFC regions is the result of stimulus, response, or mapping rules between them.

LPFC coding of only contextually relevant information dimensions is consistent with the adaptive coding model of Duncan (2001) in which PFC flexibly encodes only task-relevant information. Other empirical studies are also consistent with adaptive coding: LPFC activity for identical stimulus arrays varies with task context such that task-relevant information (target location) is preferentially encoded over task-irrelevant information (identities of all stimulus array items) (Rainer et al., 1998). Similarly, LPFC neurons make the task-relevant distinction between targets and distractors, but not between distractor exemplars (Everling et al., 2002; Kusunoki et al., 2009). Finally, LPFC activity depends upon current task-relevant stimulus categorization instead of previous categorization rules for the identical stimulus (Freedman et al., 2001; Roy et al., 2010). Such contextual encoding avoids LPFC duplication of posterior cortical representations. Given energy and space constraints on the brain, there may be evolutionary pressure to reduce duplication and increase sparseness and flexibility of neural coding (see, e.g., Attwell and Laughlin, 2001; Lennie, 2003; Olshausen and Field, 2004).

Our results are consistent with a subset of LPFC comprising the neural basis of the RS bottleneck limiting dual-task performance (Pashler, 1994; Dux et al., 2006; Tombu et al., 2011). The present findings provide a potential mechanism to account for such central bottlenecks: The inability of prefrontal regions to separately encode distinct arbitrary RS rules creates a serial bottleneck in which only one RS process can be coded and executed 
at a time. As such, these findings reveal the downside of a neural system endowed with adaptive coding: an inability to flexibly code more than one set of task-relevant information at a time.

Finally, the present study demonstrates that frontoparietal regions are heterogeneous in their ability to encode task-relevant information: different regions coded distinct task dimensions (e.g., modality vs process) to varying extents, hinting at separable roles for each region despite frequent coactivation. Furthermore, given that we found no RS region that was amodal and processinsensitive, there may be no wholly general-purpose executive region or network that uses completely abstract information codes. However, the finding that some MD regions may encode neither modality nor process (Fig. 10) does hint at possible general-purpose regions (e.g., Davelaar, 2011). Determining whether any region uses an information code indifferent to all task dimensions is an important issue that is ripe for future experimentation.

\section{References}

Artchakov D, Tikhonravov D, Vuontela V, Linnankoski I, Korvenoja A, Carlson S (2007) Processing of auditory and visual location information in the monkey prefrontal cortex. Exp Brain Res 180:469-479. CrossRef Medline

Asaad WF, Rainer G, Miller EK (2000) Task-specific neural activity in the primate prefrontal cortex. J Neurophysiol 84:451-459. Medline

Asplund CL (2010) The coordination and control of attention in lateral prefrontal cortex. Doctoral dissertation. In: Neuroscience. Nashville, TN: Vanderbilt University.

Attwell D, Laughlin SB (2001) An energy budget for signaling in the grey matter of the brain. J Cereb Blood Flow Metab 21:1133-1145. CrossRef Medline

Avants BB, Tustison NJ, Song G, Cook PA, Klein A, Gee JC (2011) A reproducible evaluation of ANTs similarity metric performance in brain image registration. Neuroimage 54:2033-2044. CrossRef Medline

Baddeley A (2003) Working memory: looking back and looking forward. Nat Rev Neurosci 4:829-839. CrossRef Medline

Baddeley A, Hitch GJ (1974) Working memory. In: The psychology of learning and motivation (Bower GH, ed), pp 47-89. New York: Academic.

Badre D (2008) Cognitive control, hierarchy, and the rostro-caudal organization of the frontal lobes. Trends Cogn Sci 12:193-200. CrossRef Medline

Badre D, D'Esposito M (2009) Is the rostro-caudal axis of the frontal lobe hierarchical? Nat Rev Neurosci 10:659-669. CrossRef Medline

Birn RM, Cox RW, Bandettini PA (2004) Experimental designs and processing strategies for fMRI studies involving overt verbal responses. Neuroimage 23:1046-1058. CrossRef Medline

Bode S, Haynes JD (2009) Decoding sequential stages of task preparation in the human brain. Neuroimage 45:606-613. CrossRef Medline

Boynton GM, Engel SA, Glover GH, Heeger DJ (1996) Linear systems analysis of functional magnetic resonance imaging in human V1. J Neurosci 16:4207-4221. Medline

Brainard DH (1997) The psychophysics toolbox. Spat Vis 10:433-436. CrossRef Medline

Chang CC, Lin CJ (2011) LIBSVM: a library for support vector machines. ACM Trans Intell Syst Technol 2:1-27. CrossRef

Christophel TB, Hebart MN, Haynes JD (2012) Decoding the contents of visual short-term memory from human visual and parietal cortex. J Neurosci 32:12983-12989. CrossRef Medline

Cole MW, Etzel JA, Zacks JM, Schneider W, Braver TS (2011) Rapid transfer of abstract rules to novel contexts in human lateral prefrontal cortex. Front Hum Neurosci 5:142. CrossRef Medline

Courtney SM (2004) Attention and cognitive control as emergent properties of information representation in working memory. Cogn Affect Behav Neurosci 4:501-516. CrossRef Medline

Cowan N, Li D, Moffitt A, Becker TM, Martin EA, Saults JS, Christ SE (2011) A neural region of abstract working memory. J Cogn Neurosci 23:28522863. CrossRef Medline

Cromer JA, Roy JE, Miller EK (2010) Representation of multiple, indepen- dent categories in the primate prefrontal cortex. Neuron 66:796-807. CrossRef Medline

Davelaar EJ (2011) Processes versus representations: cognitive control as emergent, yet componential. Top Cogn Sci 3:247-252. CrossRef

Desimone R, Duncan J (1995) Neural mechanisms of selective visualattention. Annu Rev Neurosci 18:193-222. CrossRef Medline

Duncan J (2001) An adaptive coding model of neural function in prefrontal cortex. Nat Rev Neurosci 2:820-829. CrossRef Medline

Duncan J (2010) The multiple-demand (MD) system of the primate brain: mental programs for intelligent behaviour. Trends Cogn Sci 14:172-179. CrossRef Medline

Duncan J, Owen AM (2000) Common regions of the human frontal lobe recruited by diverse cognitive demands. Trends Neurosci 23:475-483. CrossRef Medline

Dux PE, Ivanoff J, Asplund CL, Marois R (2006) Isolation of a central bottleneck of information processing with time-resolved fMRI. Neuron 52: 1109-1120. CrossRef Medline

Dux PE, Tombu MN, Harrison S, Rogers BP, Tong F, Marois R (2009) Training improves multitasking performance by increasing the speed of information processing in human prefrontal cortex. Neuron 63:127-138. CrossRef Medline

Esterman M, Chiu YC, Tamber-Rosenau BJ, Yantis S (2009) Decoding cognitive control in human parietal cortex. Proc Natl Acad Sci U S A 106: 17974-17979. CrossRef Medline

Etzel JA, Valchev N, Keysers C (2011) The impact of certain methodological choices on multivariate analysis of fMRI data with support vector machines. Neuroimage 54:1159-1167. CrossRef Medline

Everling S, Tinsley CJ, Gaffan D, Duncan J (2002) Filtering of neural signals by focused attention in the monkey prefrontal cortex. Nat Neurosci 5:671-676. CrossRef Medline

Fox MD, Snyder AZ, Vincent JL, Corbetta M, Van Essen DC, Raichle ME (2005) The human brain is intrinsically organized into dynamic, anticorrelated functional networks. Proc Natl Acad Sci U S A 102:9673-9678. CrossRef Medline

Freedman DJ, Riesenhuber M, Poggio T, Miller EK (2001) Categorical representation of visual stimuli in the primate prefrontal cortex. Science 291:312-316. CrossRef Medline

Friston KJ, Frith CD, Turner R, Frackowiak RS (1995) Characterizing evoked hemodynamics with fMRI. Neuroimage 2:157-165. CrossRef Medline

Fuster JM (2001) The prefrontal cortex-an update: time is of the essence. Neuron 30:319-333. CrossRef Medline

Gao W, Lin W (2012) Frontal parietal control network regulates the anticorrelated default and dorsal attention networks. Hum Brain Mapp 33: 192-202. CrossRef Medline

Huang J, Francis AP, Carr TH (2008) Studying overt word reading and speech production with event-related fMRI: a method for detecting, assessing, and correcting articulation-induced signal changes and for measuring onset time and duration of articulation. Brain Lang 104:10-23. CrossRef Medline

Ivanoff J, Branning P, Marois R (2009) Mapping the pathways of information processing from sensation to action in four distinct sensorimotor tasks. Hum Brain Mapp 30:4167-4186. CrossRef Medline

Jiang Y, Kanwisher N (2003) Common neural substrates for response selection across modalities and mapping paradigms. J Cogn Neurosci 15: 1080-1094. CrossRef Medline

Kamienkowski JE, Pashler H, Dehaene S, Sigman M (2011) Effects of practice on task architecture: combined evidence from interference experiments and random-walk models of decision making. Cognition 119:81-95. CrossRef Medline

Kamitani Y, Sawahata Y (2010) Spatial smoothing hurts localization but not information: pitfalls for brain mappers. Neuroimage 49:1949-1952. CrossRef Medline

Kamitani Y, Tong F (2005) Decoding the visual and subjective contents of the human brain. Nat Neurosci 8:679-685. CrossRef Medline

Keysers C, Kohler E, Umiltà MA, Nanetti L, Fogassi L, Gallese V (2003) Audiovisual mirror neurons and action recognition. Exp Brain Res 153: 628-636. CrossRef Medline

Kleiner M, Brainard D, Pelli D (2007) What's new in Psychtoolbox-3? Perception 36:14.

Koechlin E, Ody C, Kouneiher F (2003) The architecture of cognitive con- 
trol in the human prefrontal cortex. Science 302:1181-1185. CrossRef Medline

Kriegeskorte N, Simmons WK, Bellgowan PS, Baker CI (2009) Circular analysis in systems neuroscience: the dangers of double dipping. Nat Neurosci 12:535-540. CrossRef Medline

Kusunoki M, Sigala N, Gaffan D, Duncan J (2009) Detection of fixed and variable targets in the monkey prefrontal cortex. Cereb Cortex 19:25222534. CrossRef Medline

Lennie P (2003) The cost of cortical computation. Curr Biol 13:493-497. CrossRef Medline

Levy R, Goldman-Rakic PS (2000) Segregation of working memory functions within the dorsolateral prefrontal cortex. Exp Brain Res 133:23-32. CrossRef Medline

Marois R, Larson JM, Chun MM, Shima D (2006) Response-specific sources of dual-task interference in human pre-motor cortex. Psychol Res 70:436-447. CrossRef Medline

Meyers EM, Freedman DJ, Kreiman G, Miller EK, Poggio T (2008) Dynamic population coding of category information in inferior temporal and prefrontal cortex. J Neurophysiol 100:1407-1419. CrossRef Medline

Nee DE, Brown JW, Askren MK, Berman MG, Demiralp E, Krawitz A, Jonides J (2013) A meta-analysis of executive components of working memory. Cereb Cortex 23:264-282. CrossRef Medline

Nichols T, Brett M, Andersson J, Wager T, Poline JB (2005) Valid conjunction inference with the minimum statistic. Neuroimage 25:653-660. CrossRef Medline

Niendam TA, Laird AR, Ray KL, Dean YM, Glahn DC, Carter CS (2012) Meta-analytic evidence for a superordinate cognitive control network subserving diverse executive functions. Cogn Affect Behav Neurosci 12: 241-268. CrossRef Medline

Norman DA, Bobrow DG (1975) On data-limited and resource-limited processes. Cogn Psychol 7:44-64. CrossRef

Norman DA, Shallice T (1986) Attention to action: willed and automatic control of behaviour. In: Consciousness and self-regulation: advances in research and theory (Davidson RJ, Schwarts GE, Shapiro D, eds), pp 1-18. New York: Plenum.

Olshausen BA, Field DJ (2004) Sparse coding of sensory inputs. Curr Opin Neurobiol 14:481-487. CrossRef Medline

Op de Beeck HP (2010a) Probing the mysterious underpinnings of multivoxel fMRI analyses. Neuroimage 50:567-571. CrossRef Medline

Op de Beeck HP (2010b) Against hyperacuity in brain reading: spatial smoothing does not hurt multivariate fMRI analyses? Neuroimage 49: 1943-1948. CrossRef Medline

Pashler H (1994) Dual-task interference in simple tasks: data and theory. Psychol Bull 116:220-244. CrossRef Medline

Pashler H, Carrier M, Hoffman J (1993) Saccadic eye movements and dualtask interference. Q J Exp Psychol A 46:51-82. Medline

Pelli DG (1997) The VideoToolbox software for visual psychophysics: transforming numbers into movies. Spat Vis 10:437-442. CrossRef Medline

Power JD, Barnes KA, Snyder AZ, Schlaggar BL, Petersen SE (2012) Spurious but systematic correlations in functional connectivity MRI networks arise from subject motion. Neuroimage 59:2142-2154. CrossRef Medline

Rainer G, Asaad WF, Miller EK (1998) Selective representation of relevant information by neurons in the primate prefrontal cortex. Nature 393:577-579. CrossRef Medline

Raj D, Paley DP, Anderson AW, Kennan RP, Gore JC (2000) A model for susceptibility artefacts from respiration in functional echo-planar magnetic resonance imaging. Phys Med Biol 45:3809-3820. CrossRef Medline

Raj D, Anderson AW, Gore JC (2001) Respiratory effects in human functional magnetic resonance imaging due to bulk susceptibility changes. Phys Med Biol 46:3331-3340. CrossRef Medline

Reverberi C, Gorgen K, Haynes JD (2012) Compositionality of rule representations in human prefrontal cortex. Cereb Cortex 32:1237-1246. CrossRef Medline

Riggall AC, Postle BR (2012) The relationship between working memory storage and elevated activity as measured with functional magnetic resonance imaging. J Neurosci 32:12990-12998. CrossRef Medline
Rouder JN, Morey RD (2011) A Bayes factor meta-analysis of Bem's ESP claim. Psychon Bull Rev 18:682-689. CrossRef Medline

Rouder JN, Speckman PL, Sun D, Morey RD, Iverson G (2009) Bayesian $t$ tests for accepting and rejecting the null hypothesis. Psychon Bull Rev 16:225-237. CrossRef Medline

Roy JE, Riesenhuber M, Poggio T, Miller EK (2010) Prefrontal cortex activity during flexible categorization. J Neurosci 30:8519-8528. CrossRef Medline

Sabuncu MR, Singer BD, Conroy B, Bryan RE, Ramadge PJ, Haxby JV (2010) Function-based intersubject alignment of human cortical anatomy. Cereb Cortex 20:130-140. CrossRef Medline

Sala JB, Rämä P, Courtney SM (2003) Functional topography of a distributed neural system for spatial and nonspatial information maintenance in working memory. Neuropsychologia 41:341-356. CrossRef Medline

Sathian K, Lacey S, Stilla R, Gibson GO, Deshpande G, Hu X, Laconte S, Glielmi C (2011) Dual pathways for haptic and visual perception of spatial and texture information. Neuroimage 57:462-475. CrossRef Medline

Schumacher EH, Schwarb H, Lightman E, Hazeltine E (2011) Investigating the modality specificity of response selection using a temporal flanker task. Psychol Res 75:499-512. CrossRef Medline

Shomstein S, Yantis S (2004) Control of attention shifts between vision and audition in human cortex. J Neurosci 24:10702-10706. CrossRef Medline

Szameitat AJ, Schubert T, Müller K, Von Cramon DY (2002) Localization of executive functions in dual-task performance with fMRI. J Cogn Neurosci 14:1184-1199. CrossRef Medline

Talairach J, Tournoux P (1988) Co-planar stereotaxic atlas of the human brain. New York: Thieme.

Tamber-Rosenau BJ, Esterman M, Chiu YC, Yantis S (2011) Cortical mechanisms of cognitive control for shifting attention in vision and working memory. J Cogn Neurosci 23:2905-2919. CrossRef Medline

Todd JJ, Marois R (2004) Capacity limit of visual short-term memory in human posterior parietal cortex. Nature 428:751-754. CrossRef Medline

Todd JJ, Marois R (2005) Posterior parietal cortex activity predicts individual differences in visual short-term memory capacity. Cogn Affect Behav Neurosci 5:144-155. CrossRef Medline

Tombu MN, Asplund CL, Dux PE, Godwin D, Martin JW, Marois R (2011) A unified attentional bottleneck in the human brain. Proc Natl Acad Sci U S A 108:13426-13431. CrossRef Medline

Vincent JL, Kahn I, Snyder AZ, Raichle ME, Buckner RL (2008) Evidence for a frontoparietal control system revealed by intrinsic functional connectivity. J Neurophysiol 100:3328-3342. CrossRef Medline

Warden MR, Miller EK (2007) The representation of multiple objects in prefrontal neuronal delay activity. Cereb Cortex 17 [suppl 1]:i41-i50.

Warden MR, Miller EK (2010) Task-dependent changes in short-term memory in the prefrontal cortex. J Neurosci 30:15801-15810. CrossRef Medline

Watanabe M (1992) Frontal units of the monkey coding the associative significance of visual and auditory stimuli. Exp Brain Res 89:233-247. Medline

Wetzels R, Matzke D, Lee MD, Rouder JN, Iverson GJ, Wagenmakers EJ (2011) Statistical evidence in experimental psychology: an empirical comparison using $855 t$ tests. Perspect Psychol Sci 6:291-298. CrossRef

Wollberg Z, Sela J (1980) Frontal-cortex of the awake squirrel-monkeyresponses of single cells to visual and auditory-stimuli. Brain Res 198: 216-220. CrossRef Medline

Woolgar A, Thompson R, Bor D, Duncan J (2011a) Multi-voxel coding of stimuli, rules, and responses in human frontoparietal cortex. Neuroimage 56:744-752. CrossRef Medline

Woolgar A, Hampshire A, Thompson R, Duncan J (2011b) Adaptive coding of task-relevant information in human frontoparietal cortex. J Neurosci 31:14592-14599. CrossRef Medline

Yarkoni T (2009) Big correlations in little studies: inflated fMRI correlations reflect low statistical power: commentary on Vul et al. (2009). Perspect Psychol Sci 4:294-298. CrossRef

Zylberberg A, Fernández Slezak D, Roelfsema PR, Dehaene S, Sigman M (2010) The brain's router: a cortical network model of serial processing in the primate brain. PLoS Comput Biol 6:e1000765. CrossRef Medline 